\title{
Extended deformation functors
}

\author{
Marco Manetti* \\ Università di Roma "La Sapienza", Italy
}

October 15, 2018

\begin{abstract}
We introduce a precise notion, in terms of some Schlessinger's type conditions, of extended deformation functors which is compatible with most of recent ideas in the Derived Deformation Theory (DDT) program and with geometric examples. With this notion we develop the (extended) analogue of Schlessinger and obstruction theories. The inverse mapping theorem holds for natural transformations of extended deformation functors and all such functors with finite dimensional tangent space are prorepresentable in the homotopy category. Finally we prove that the primary obstruction map induces a structure of graded Lie algebra on the tangent space.
\end{abstract}

Mathematics Subject Classification (1991): 13D10, 14B10, 14 D15.

\section{Introduction}

This paper is devoted to the study of some foundations in the derived deformation theory (DDT) program, see [幽, [1].

One of the main aspects of DDT program is the Hidden Smoothness Philosophy: this is based on the idea that, in characteristic 0 , every "reasonable" deformation problem give rise naturally to an extended "quasismooth" differential $\mathbb{Z}$-graded moduli space $\mathcal{M}^{\mathbb{Z}}$ such that the ordinary moduli space $\mathcal{M}$ is just the truncation in degree 0 of $\mathcal{M}^{\mathbb{Z}}$. Roughly speaking a differential $\mathbb{Z}$-graded space is a ringed space whose structure sheaf takes value in differential $\mathbb{Z}$-graded connected algebras; see [4], [11] for details. In its first written reference [15], the DDT program was proposed as a possible way to define virtual tangent bundle and virtual fundamental class on moduli spaces, while more recent applications of extended moduli spaces [1], [2], concern the understanding of mirror symmetry for Calabi-Yau manifolds.

The hidden smoothness philosophy applied to infinitesimal deformation theory should implies that, over a field of characteristic 0, every reasonable deformation functor is the truncation of a suitable quasismooth extended deformation functor. Formally a functor $F$ from the category $\mathbf{A r t}_{\mathbb{K}}$ of local Artinian $\mathbb{K}$-algebras with residue field $\mathbb{K}$ to the category of sets is called a deformation functor if it satisfies the (slightly modified, see the discussion in [6], [7] and references therein) Schlessinger's conditions

1) $F(\mathbb{K})=\{0\}$.

2) $F\left(A \times_{\mathbb{K}} B\right)=F(A) \times F(B)$.

3) The map $F\left(A \times_{C} B\right) \rightarrow F(A) \times_{F(C)} F(B)$ is surjective for every pair of morphism $\alpha: A \rightarrow C, \beta: B \rightarrow C$ with $\alpha$ surjective.

\footnotetext{
*partially supported by Italian MURST program 'Spazi di moduli e teoria delle rappresentazioni'. Member of GNSAGA of CNR.

${ }^{1}$ Some authors prefer to say "smooth in an appropriate sense"
} 
and the term "reasonable" means essentially that $F$ arises from a geometric deformation problem.

By an extended deformation functor we understand a set valued functor $F$ defined on a category $\mathbf{C}$ containing $\mathbf{A r t}_{\mathbb{K}}$ as a full subcategory and such that $F$ satisfies some extended Schlessinger's conditions.

As far as we known there are in literature two main notions of extended deformation functors.

The first ([1], [2]) considers $\mathbf{C}$ as the category of $\mathbb{Z}$-graded local Artinian $\mathbb{K}$-algebras with residue field $\mathbb{K}$ and the extended Schlessinger's conditions are nothing else than the trivial extension of 1), 2) and 3). This approach does not present any additional difficulty with respect to the classical case but works well only when the associated extended moduli space is smooth in the (strong) sense of [1, 2.2].

The second approach ([13], [4] $)$, takes $\mathbf{C}$ as the category of differential $\mathbb{Z}$-graded local Artinian $\mathbb{K}$-algebras with residue field $\mathbb{K}$ that are homotopy equivalent to algebras concentrated in nonpositive degrees and the generalized Schlessinger condition are the above 1), 2), 3) together with the following:

4) $F$ sends quasiisomorphisms in $\mathbf{C}$ into invertible maps in Set.

This fourth condition is the principal responsible of some hidden smoothness phenomena of extended deformation functors. This approach works usually quite well for the extension of (classical) prorepresentable deformation functors, e.g. the Hilbert functor, but fails to be good in more general situations (see the discussion in [16, 3.3]).

In this paper we propose a notion of extended deformation functor which contains the previous two as specializations. In doing that we always keep in mind two general ideas: the first is taken from [16], while the second is nowadays standard.

a) $\mathbf{C}$ is the category of all differential $\mathbb{Z}$-graded associative (graded)-commutative local Artinian $\mathbb{K}$-algebras with residue field $\mathbb{K}$.

b) Over a field of characteristic 0, every reasonable deformation problem is governed by a differential graded Lie algebra; therefore the functor of Maurer-Cartan solutions of a differential graded Lie algebra modulo gauge action must be considered as the basic example of deformation functor.

Some easy examples show that the above condition 4) is not compatible with a) and b); this is one of the motivation of this paper and forces us to give a more technical definition of deformation functor, see 2.1. The paper goes as follows:

In Section 1 we recall some definitions from rational homotopy theory and we fix the notation for the rest of the paper.

In Section 2 we propose a precise definition of deformation functors in terms of some Schlessinger's type conditions and we show that our notion is compatible with the above ideas a) and b). As in the classical case, every deformation functor $F$ has a tangent space $T F[1]$ which is a $\mathbb{Z}$-graded vector space. For notational convenience we always replace local Artinian rings with their maximal ideals; this means that, in our description, $\mathbf{C}$ is the category of finite dimensional associative commutative $\mathbb{Z}$-graded nilpotent differential $\mathbb{K}$-algebras.

In Section 3 we show that the inverse function theorem holds for morphisms of deformation functors (Cor. 3.3); this implies in particular a conceptually easier proof of the well known fact that every quasiisomorphism of differential graded Lie algebras induces an isomorphisms of deformation functors. We also prove that every deformation functor has a natural complete obstruction theory with obstruction space $T F[2]$ (by definition $T F[2]$ is a graded vector space with $\left.T F[2]^{i}=T F[1]^{i+1}\right)$.

\footnotetext{
${ }^{2}$ From now on we always omit the term 'extended' before 'deformation functor'
} 
In Section 4 we generalize the main result of [23]; every deformation functor $F$ induces naturally a functor $[F]$ defined in the homotopy category $K(\mathbf{C})$ (see 1.2). Theorem 4.5 asserts that, if the tangent space $T F[1]$, is finite dimensional then $[F]$ is prorepresented by a pronilpotent differential algebra $(R, d)$, where $R$ is the inverse limit of $R / R^{n+1}=\oplus_{i=1}^{n}\left(\odot^{i} T F[1]^{\vee}\right)$ and $d$ is a differential of degree +1 .

In Section 5 we will deal with extended deformation functors in the set-up of dg-coalgebras and we introduce the deformation functors of a $L_{\infty}$-algebra.

In Section 6 we will prove the existence of a natural graded Lie algebra structure over the (shifted) tangent space of a deformation functor.

In Section 7 we give a necessary and sufficient condition, always satisfied in concrete examples, for a deformation functor to be isomorphic to the deformation functor of a $L_{\infty}$ algebra.

This paper owes its existence to the participation of the author to the "Deformation quantization seminar", held in Scuola Normale Superiore di Pisa during the academic year 1998-99. It is a pleasure to thank here E. Arbarello, G. Bini, A. Canonaco, P. de Bartolomeis, F. de Vita, D. Fiorenza, G. Gaiffi, M. Grassi, M. Polito and R. Silvotti for useful and stimulating discussions.

\section{Notation}

We will always work over a fixed field $\mathbb{K}$ of characteristic 0 . All vector spaces, linear maps, algebras, tensor products, derivations etc.. are understood of being over $\mathbb{K}$, unless otherwise specified.

By $\Sigma_{n}$ we denote the symmetric group of permutations of $\{1, \ldots, n\}$. By $S(p, q) \subset \Sigma_{p+q}$ we denote the set of unshuffles of type $(p, q)$; by definition $\sigma \in S(p, q)$ if and only if $\sigma(i)<\sigma(i+1)$ for every $i \neq p$. The unshuffles are a set of representative for the cosets of $\Sigma_{p} \times \Sigma_{q}$ inside $\Sigma_{p+q}$.

\section{Differential graded algebras and homotopy}

We denote by: Set the category of sets in a fixed universe; $\mathbf{G}$ the category of $\mathbb{Z}$-graded vector spaces. If $V$ is a graded vector space and $v \in V, v \neq 0$, is a homogeneous element, we denote by $\bar{v} \in \mathbb{Z}$ its degree.

DG denotes the category of complexes of vector spaces, also called differential graded vector spaces. Every object in DG can be considered as a pairf $(V, d)$, where $V$ is an object in $\mathbf{G}$ and the differential $d: V \rightarrow V$ is a linear map such that $d\left(V^{i}\right) \subset V^{i+1}$ and $d^{2}=0$. We will consider $\mathbf{G}$ as the full subcategory of $\mathbf{D G}$ whose objects are the complexes with zero differential.

For every complex of vector spaces $V \in \mathbf{D G}$ we denote, as usual, by $Z^{*}(V), B^{*}(V)$ and $H^{*}(V)$ the cocycles, coboundaries and cohomology of $V$.

The tensor product in the category DG is defined in the standard way

$$
(V \otimes W)^{i}=\oplus_{j \in \mathbb{Z}} V^{j} \otimes W^{i-j}, \quad d(v \otimes w)=d v \otimes w+(-1)^{\bar{v}} v \otimes d w
$$

The twisting map $T: V \otimes W \rightarrow W \otimes V$ is defined by $T(x \otimes y)=(-1)^{\bar{x}} \bar{y} y \otimes x$ and then extended by linearity; $T$ is a morphism of complexes and define a $\mathbb{Z} / 2 \mathbb{Z}$-action on $V \otimes V$.

\footnotetext{
${ }^{3}$ For notational convenience, when the risk of confusion is remote, we prefer to write $V$ for the complex omitting the differential; the same will be made for all algebraic structures builded over a graded vector space.
} 
This action extends naturally to an action of the symmetric group $\Sigma_{n}$ on $\otimes^{n} V$. The Koszul $\operatorname{sign} \epsilon\left(\sigma ; v_{1}, \ldots, v_{n}\right)= \pm 1, \sigma \in \Sigma_{n}$, is defined by

$$
\sigma\left(v_{1} \otimes \ldots \otimes v_{n}\right)=\epsilon\left(\sigma ; v_{1}, \ldots, v_{n}\right)\left(v_{\sigma(1)} \otimes \ldots \otimes v_{\sigma(n)}\right) .
$$

In the next formulas we simply write $\epsilon(\sigma)$ instead of $\epsilon\left(\sigma ; v_{1}, \ldots, v_{n}\right)$ when there is no ambiguity about $v_{1}, \ldots, v_{n} \in V$.

The symmetric power $\odot{ }^{n} V$ is the quotient of $\otimes^{n} V$ by the subspace generated by the elements $v_{1} \otimes \ldots \otimes v_{n}-\sigma\left(v_{1} \otimes \ldots \otimes v_{n}\right)$.

Given an integer $n$, the shift functor $[n]: \mathbf{D G} \rightarrow \mathbf{D G}$ is defined by setting $V[n]=$ $\mathbb{K}[n] \otimes V, f[n]=I d_{\mathbb{K}[n]} \otimes f$, where

$$
\mathbb{K}[n]_{i}= \begin{cases}\mathbb{K} & \text { if } i+n=0 \\ 0 & \text { otherwise }\end{cases}
$$

Given $v \in V$ we also denote by $v[n]=1_{\mathbb{K}[n]} \otimes v \in V[n]$, where $1_{\mathbb{K}[n]}$ is the unity of $\mathbb{K}$ shifted in degree $-n$; note that $\overline{v[n]}=\bar{v}-n$.

More informally, the complex $V[n]$ is the complex $V$ with indices shifted by $n$ and differential multiplied by $(-1)^{n}$. Note that $\operatorname{Hom}_{\mathbf{D G}}(V, W[n])$ are the linear maps $f: V \rightarrow W$ such that $f\left(V_{i}\right) \subset W_{i+n}$ for every $i$ and $f d_{V}=d_{W[n]} f=(-1)^{n} d_{W} f$.

A commutative, associative graded algebra is the data of a graded vector space $A \in \mathbf{G}$ together an associative, linear multiplication map : $A \otimes A \longrightarrow A$ such that $A_{i} A_{j} \subset A_{i+j}$ and $a b=(-1)^{\bar{a}} \bar{b} b a$.

We denote by GA the category of (commutative, associative) graded algebras. A graded algebra $A$ is called nilpotent if $A^{n}=0$ for $n>>0$; clearly every nilpotent algebra is without unit.

A commutative associative differential graded algebra (dg-algebra for short) is an object $(A, d) \in \mathbf{D G}$ such that $A$ is a graded algebras and $d$ is a derivation of degree 1 . This means that $d$ satisfies the (graded) Leibnitz rule $d(a b)=d(a) b+(-1)^{\bar{a}} a d(b)$. If $A$ has a unit 1 , we assume moreover that $1 \notin d(A)$.

We denote by DGA the category of dg-algebras. In the sequel of the paper we consider DG and GA as the full subcategories of DGA whose object are respectively the dg-algebras with trivial multiplication and with trivial differential. We also denote by NDGA the full subcategory of nilpotent dg-algebras and by $\mathbf{C}$ the full subcategory of nilpotent dg-algebras which are finite dimensional as $\mathbb{K}$-vector space. Note that in our convention $\mathbf{D G} \cap \mathbf{C}$ denotes the dg-algebras $A \in \mathbf{C}$ with trivial multiplication.

Example 1.1. If $A \in \mathbf{C}$, then $\mathbb{K} \oplus A$ is a local Artinian ring; conversely it is easy to see that every local Artinian dg-algebra with unit and residue field $\mathbb{K}$ has the form $\mathbb{K} \oplus A$ for some $A \in \mathbf{C}$

A module over a dg-algebra $A$ is the data of a complex $M \in \mathbf{D G}$ together two associative multiplication maps $A \otimes M \rightarrow M$ (left multiplication), $M \otimes A \rightarrow M$ (right multiplication) which are morphisms in DG commuting with the twisting map. This means that:

- $a m=(-1)^{\bar{a} \bar{m}} m a$ for every homogeneous $a \in A, m \in M$.

- $d_{M}(a m)=d_{A}(a) m+(-1)^{\bar{a}} a d_{M}(m), d_{M}(m a)=d_{M}(m) a+(-1)^{\bar{m}} m d_{A}(a)$.

If $A$ has a unit 1 we also assume that $1 \cdot m=m \cdot 1=m$.

If $M$ is an $A$-module and $n \in \mathbb{Z}$ then there is a natural structure of $A$-module over $M[n]$ having the same right multiplication and the left multiplication induced via the twisting map $T$.

${ }^{4}$ Here we follows the notation of [16]; in $[22]$ the complex $V[-n]$ is called the ' $n$-fold suspension of $V$ ' 
A derivation of a dg-algebra $A$ into an $A$-module $M$ is a morphism of graded vector spaces $h: A \rightarrow M$ which satisfy the Leibnitz rule $h(a b)=h(a) b+a h(b)$. We denote by $\operatorname{Der}(A, M)$ the $A_{0}$-module of derivations $h: A \rightarrow M$. Notice that the differential of $A$ is an element of $\operatorname{Der}(A, A[1])$ and that if $A^{2}=A M=0$ then $\operatorname{Der}(A, M)$ is the space of morphisms in the category $\mathbf{G}$ from $A$ to $M$.

In the above set-up it is also defined an $A$-module $\operatorname{Der}^{*}(A, M) \in \mathbf{D G}$ with $\operatorname{Der}^{n}(A, M)=$ $\operatorname{Der}(A, M[n])$, the obvious left multiplication and differential

$$
\delta: \operatorname{Der}(A, M[n]) \rightarrow \operatorname{Der}(A, M[n+1]) ; \quad \delta(h)=d_{M[n]} h-h d_{A}
$$

Given a morphism of $A$-modules $f: M \rightarrow A$ such that $f(M) M=0$ (in most applications $M$ will be a square-zero ideal of the dg-algebra $A$ and $f$ the inclusion) we define the mapping cone as the dg-algebra $C=A \oplus M[1]$ with the product $(a, m)(b, n)=(a b, a n+m b)$ (note that, as a graded algebra, $C$ is the trivial extension of $A$ by $M[1])$ and differential

$$
d_{C}=\left(\begin{array}{cc}
d_{A} & f \\
0 & d_{M[1]}
\end{array}\right): A \oplus M[1] \rightarrow A[1] \oplus M[2]
$$

The reader must be careful here: the product in $C$ is defined using the $A$-module structure of $M[1]$. We left to the reader the easy verification that the mapping cone $C$ is a dg-algebra, the inclusion $A \rightarrow C$ is a morphism of dg-algebras and the projection $C \rightarrow M[1]$ is a derivation.

Conversely, given a derivation $h: B \rightarrow N$ we define the derived inverse mapping cone as the dg-algebra $D=B \oplus N[-1]$ with product $(a, m)(b, n)=(a b, a n+m b)$ and differential

$$
d_{D}=\left(\begin{array}{cc}
d_{B} & 0 \\
h & d_{N[-1]}
\end{array}\right): B \oplus N[-1] \rightarrow B[1] \oplus N
$$

Here the projection $D \rightarrow B$ is a morphism of dg-algebras and the inclusion $N[-1] \rightarrow D$ is a morphism of $D$-modules.

We denote by $\mathbb{K}\left[t_{1}, \ldots, t_{n}, d t_{1}, \ldots, d t_{n}\right]$ the dg-algebra of polynomial differential forms on the affine space $\mathbb{A}^{n}$ with the de Rham differential. We have $\mathbb{K}[t, d t]=\mathbb{K}[t] \oplus \mathbb{K}[t] d t$ and

$$
\mathbb{K}\left[t_{1}, \ldots, t_{n}, d t_{1}, \ldots, d t_{n}\right]=\otimes_{i=1}^{\otimes} \mathbb{K}\left[t_{i}, d t_{i}\right]
$$

Since $\mathbb{K}$ has characteristic 0 , it is immediate to see that $H_{*}(\mathbb{K}[t, d t])=\mathbb{K}[0]$ and then by Künneth formula $H_{*}\left(\mathbb{K}\left[t_{1}, \ldots, t_{n}, d t_{1}, \ldots, d t_{n}\right]\right)=\mathbb{K}[0]$.

Note that for every dg-algebra $A$ and every $s=\left(s_{1}, \ldots, s_{n}\right) \in \mathbb{K}^{n}$ we have an evaluation morphism $e_{s}: A \otimes \mathbb{K}\left[t_{1}, \ldots, t_{n}, d t_{1}, \ldots, d t_{n}\right] \rightarrow A$ defined by

$$
e_{s}\left(a \otimes p\left(t_{1}, \ldots, t_{n}, d t_{1}, \ldots, d t_{n}\right)\right)=p\left(s_{1}, \ldots, s_{n}, 0, \ldots, 0\right) a
$$

For every dg-algebra $A$ we denote $A[t, d t]=A \otimes \mathbb{K}[t, d t]$; if $A$ is nilpotent then $A[t, d t]$ is still nilpotent. In the next sections the obvious fact that $A[t, d t]$ does not belong to $\mathbf{C}$ for every $A \neq 0$ will cause some problems whose solution is the introduction, for every positive real number $\epsilon>0$ of the dg-subalgebra

$$
A[t, d t]_{\epsilon}=A \oplus \oplus_{n>0} A^{\lceil n \epsilon\rceil} \otimes\left(\mathbb{K} t^{n} \oplus \mathbb{K} t^{n-1} d t\right) \subset A[t, d t]
$$

It is clear that if $A \in \mathbf{C}$ then $A[t, d t]_{\epsilon} \in \mathbf{C}$ for every $\epsilon>0$ and $A[t, d t]$ is the union of all $A[t, d t]_{\epsilon}, \epsilon>0$.

Definition 1.2. Given two morphisms of dg-algebras $f, g: A \rightarrow B$, a homotopy between $f$ and $g$ is a morphism $H: A \rightarrow B[t, d t]$ such that $H_{0}:=e_{0} \circ H=f, H_{1}:=e_{1} \circ H=g$ (cf. 19, p. 120]).

We denote by $[A, B]$ the quotient of $\operatorname{Hom}_{\mathbf{D G}}(A, B)$ by the equivalence relation generated 
by homotopy. Since composition respects homotopy equivalence we can also consider the homotopy categories $K(\mathbf{D G A}), K(\mathbf{N D G A})$ and $K(\mathbf{C})$; it is convenient to think of $K(\mathbf{D G A})$ as the category with the same objects of DGA and with morphisms $\operatorname{Mor}(A, B)=[A, B]$. A morphism in DGA (resp.: NDGA, C) is called a homotopy equivalence if it gives an isomorphism in the corresponding homotopy category.

Two homotopic morphisms induce the same morphism in homology, see 12, p. 120]. If $A, B \in \mathbf{D G}$, then two morphisms $f, g: A \rightarrow B$ are homotopic in the sense of 1.2 if and only if $f$ is homotopic to $g$ in the usual sense. In particular every acyclic complex is contractible as a dg-algebra.

Definition 1.3. An extension in NDGA is a short exact sequence

$$
0 \longrightarrow I \longrightarrow A \stackrel{\alpha}{\longrightarrow} B \longrightarrow 0
$$

such that $\alpha$ is a morphism in NDGA and $I$ is an ideal of $A$ such that $I^{2}=0$. The extension (1) is called small if $A I=0$, it is called acyclic small if it is small and $I$ is an acyclic complex, or equivalently if $\alpha$ is a quasiisomorphism.

The easy proof of the following facts is left to the reader:

- Every surjective morphism $A \stackrel{\alpha}{\longrightarrow} B$ in the category $\mathbf{C}$ is the composition of a finite number of small extensions.

- If $A \stackrel{\alpha}{\longrightarrow} B$ is a surjective quasiisomorphism in $\mathbf{C}$ and $A_{i}=0$ for every $i>0$ then $\alpha$ is the composition of a finite number of acyclic small extensions. This is generally false if $A_{i} \neq 0$ for some $i>0$.

- For every morphism $\alpha: A \rightarrow B$ in $\mathbf{C}$ there exists a surjective homotopy equivalence $\gamma: C \rightarrow A$ in $\mathbf{C}$ such that $\alpha \gamma$ is homotopic to a surjective map. If moreover $A_{i}=B_{i}=0$ for every $i>0$ and $\alpha: H_{0}(A) \rightarrow H_{0}(B)$ is surjective then it is possible to choose $C$ such that $C_{i}=0$ for every $i>0$.

- Let $F: K(\mathbf{C}) \rightarrow$ Set be a functor such that $F(\alpha)$ is bijective for every acyclic small extension $\alpha$; then for every $A, B \in \mathbf{C}$ with $A_{i}=B_{i}=0$ for every $i>0$ and every quasiisomorphism $\gamma: A \rightarrow B$ the map $F(\gamma)$ is bijective.

\section{Extended deformation functors}

Definition 2.1. A covariant functor $F: \mathbf{C} \rightarrow$ Set is called a predeformation functor if the following conditions are satisfied:

1. $F(0)=\{0\}$ is the one-point set.

2. (Generalized Schlessinger's conditions): For every pair of morphisms $\alpha: A \rightarrow C, \beta: B \rightarrow$ $C$ in $\mathbf{C}$ consider the map

$$
\eta: F\left(A \times_{C} B\right) \rightarrow F(A) \times_{F(C)} F(B)
$$

Then:

(a) $\eta$ is surjective when $\alpha$ is surjective.

(b) $\eta$ is bijective when $\alpha$ is surjective and $C \in \mathbf{D G} \cap \mathbf{C}$ is acyclic. 
3. (Quasismoothness): For every acyclic small extension

$$
0 \longrightarrow I \longrightarrow A \longrightarrow B \longrightarrow 0
$$

the induced map $\rho: F(A) \rightarrow F(B)$ is surjective.

$A$ predeformation functor $F$ is called a deformation functor if the map $\rho$ defined in 3 is bijective.

The predeformation functors (resp.: deformation functors) together their natural transformations form a category which we denote by PreDef (resp.: Def). Note that definition 2.1 also makes sense for covariant functors $F:$ NDGA $\rightarrow$ Set.

Lemma 2.2. For a covariant functor $F: \mathbf{C} \rightarrow$ Set with $F(0)=\{0\}$ it is sufficient to check condition 20 of Definition 2.1 when $C=0$ and when $B=0$ separately.

Proof Follows immediately from the equality

$$
A \times_{C} B=(A \times B) \times{ }_{C} 0
$$

where $A \stackrel{\alpha}{\longrightarrow} C, B \stackrel{\beta}{\longrightarrow} C$ are as in $2 \mathrm{~b}$ of 2.1 and the fibred product on the right comes from the morphism $A \times B \rightarrow C,(a, b) \rightarrow \alpha(a)-\beta(b)$.

Proposition 2.3. Let $V$ be a graded vector space and let $(R, d)$ be a differential algebra such that, as a graded algebra, $R$ is the inverse limit of $R / R^{n+1}=\oplus_{i=1}^{n}\left(\odot^{i} V\right)$. Then the functor $h_{R}:$ NDGA $\rightarrow$ Set,

$$
h_{R}(A)=\operatorname{Hom}_{\mathbf{D G A}}\left(R / R^{n}, A\right), \quad n>>0
$$

is a predeformation functor.

Proof We have $F(0)=\{0\}$ and $F\left(A \times_{C} B\right)=F(A) \times_{F(C)} F(B)$; the only nontrivial condition to check is the surjectivity of $F(A) \rightarrow F(B)$ for every acyclic small extension $0 \rightarrow I \rightarrow A \stackrel{p}{\longrightarrow} B \rightarrow 0$. For simplicity we prove this in the particular case $d(R) \subset R^{2}$; the proof in the general case is essentially the same but more messy.

Let $\phi: R / R^{n} \rightarrow B$ be a morphism of dg-algebras, $\left\{v_{i}\right\} \subset V$ a homogeneous basis and denote $b_{i}=\phi\left(v_{i}\right)$. For every set of liftings $a_{i} \in A, p\left(a_{i}\right)=b_{i}, \overline{a_{i}}=\overline{b_{i}}$, there exists a unique morphism of graded algebras $\psi: R / R^{n+1} \rightarrow A$ such that $\psi\left(v_{i}\right)=a_{i}$; we need to prove that it is possible to choose the liftings such that $\psi\left(d v_{i}\right)=d a_{i}$ for every $i$.

We first note that, since $A I=0$ and $\psi\left(d v_{i}\right)-d a_{i} \in I$, the restriction $\psi: R^{2} / R^{n+1} \rightarrow A$ is a morphism of dg-algebras independent from the choice of the liftings $\left\{a_{i}\right\}$. In particular for every $i, d\left(\psi\left(d v_{i}\right)-d a_{i}\right)=0$ and, being $I$ acyclic, there exist $s_{i} \in I, \overline{s_{i}}=\overline{a_{i}}$, such that $\psi\left(d v_{i}\right)-d a_{i}=d s_{i}$. It is now sufficient to change $a_{i}$ with $a_{i}+s_{i}$ in order to transform $\psi$ into a morphism of differential graded algebras.

Definition 2.4. We shall call the differential algebra $R$ introduced in 2.3 algebraically free (or quasismooth) complete differential algebra. We also say that it is complete semifree (or semismooth) if there exists a filtration $0=H^{0} \subset H^{1} \subset \ldots \subset V$ of graded vector spaces such that $\cup_{i=0}^{\infty} H^{i}=V$ and $d\left(H^{i+1}\right) \subset \lim \oplus_{j=1}^{n}\left(\odot^{j} H^{i}\right)$.

We shall say in addition that $R$ is minimal if $d(R) \subset R^{2}$. Note that, as a graded vector space, $V=R / R^{2}$.

The terms complete in 2.4 is because $R$ is complete for the $R$-adic topology.

\section{Examples 2.5.}


1. If $R$ is a complete semifree algebra, it is easy to see that for every surjective quasiisomorphism $A \rightarrow B$ of nilpotent dg-algebras the morphism $h_{R}(A) \rightarrow h_{R}(B)$ is surjective. This property is generally false if $R$ is assumed algebraically free.

2. Let $(R, d)$ be a complete quasismooth differential algebra, $V=R / R^{2}$, then $R$ is semifree if either $V_{i}=0$ for every $i>0$ or $R$ is minimal, $V_{1}=0$ and $V_{j}=0$ for every $j<0$. In particular the maximal ideal of the algebra of functions of a smooth formal pointed $d g$-scheme (see [4]) is a complete semifree algebra.

Lemma 2.6. For a predeformation functor $F: \mathbf{C} \rightarrow$ Set the following conditions are equivalent:

i) $F$ is a deformation functor.

ii) $F$ induces a functor $[F]: K(\mathbf{C}) \rightarrow$ Set.

iii) If $I \in \mathbf{D G} \cap \mathbf{C}$ is acyclic then $F(I)=\{0\}$.

Proof i) $\Rightarrow$ ii) Let $H: A \rightarrow B[t, d t]$ be a homotopy, we need to prove that $H_{0}$ and $H_{1}$ induce the same morphism from $F(A)$ to $F(B)$. Since $A$ is finite-dimensional there exists $\epsilon>0$ sufficiently small such that $H: A \rightarrow B[t, d t]_{\epsilon}$; now the evaluation map $e_{0}: B[t, d t]_{\epsilon} \rightarrow B$ is a finite composition of acyclic small extensions and then, since $F$ is a deformation functor $F\left(B[t, d t]_{\epsilon}\right)=F(B)$; For every $a \in F(A)$ we have $H(a)=i H_{0}(a)$, where $i: B \rightarrow B[t, d t]_{\epsilon}$ is the inclusion and then $H_{1}(a)=e_{1} H(a)=e_{1} i H_{0}(a)=H_{0}(a)$.

ii) $\Rightarrow$ iii) In the homotopy category every acyclic complex is isomorphic to 0 .

iii) $\Rightarrow$ i) We need to prove that for every acyclic small extension

$$
0 \longrightarrow I \longrightarrow A \stackrel{\rho}{\longrightarrow} B \longrightarrow 0
$$

the map $F(A) \rightarrow F(B)$ is injective, this is done by showing that the diagonal map $F(A) \rightarrow$ $F(A) \times_{F(B)} F(A)$ is surjective; in order to prove this it is sufficient to prove that the diagonal map $A \rightarrow A \times_{B} A$ induces a surjective map $F(A) \rightarrow F\left(A \times_{B} A\right)$. We have a canonical isomorphism $\theta: A \times I \rightarrow A \times{ }_{B} A, \theta(a, x)=(a, a+x)$ which sends $A \times\{0\}$ onto the diagonal; since $F(A \times I)=F(A) \times F(I)=F(A)$ the proof is concluded.

A standard argument in Schlessinger's theory [23, 2.10] shows that for every predeformation functor $F$ and every $A \in \mathbf{C} \cap \mathbf{D G}$ there exists a natural structure of vector space on $F(A)$, where the sum and the scalar multiplication are described by the maps

$$
\begin{gathered}
A \times A \stackrel{+}{\longrightarrow} A \quad \Rightarrow \quad F(A \times A)=F(A) \times F(A) \stackrel{+}{\longrightarrow} F(A) \\
s \in \mathbb{K}, \quad A \stackrel{\cdot s}{\longrightarrow} A \quad \Rightarrow \quad F(A) \stackrel{\cdot s}{\longrightarrow} F(A)
\end{gathered}
$$

For every deformation functor $F$ and every integer $i$ we denote

$$
T^{i} F=F(\mathbb{K}[i-1]), \quad T F=\oplus_{i \in \mathbb{Z}} T^{i} F[-i]
$$

Every natural transformation $\phi: F \rightarrow G$ of deformation functors induces linear maps $T^{i} F \rightarrow T^{i} G$ and then a morphism of graded vector spaces $T F \rightarrow T G$.

Definition 2.7. Given a deformation functor $F$, the graded vector spaces $T F[1]$ and TF[2] are called respectively tangent and obstruction space of $F$.

Theorem 2.8. Let $F$ be a predeformation functor, then there exists a deformation functor $F^{+}$and a natural transformation $\eta: F \rightarrow F^{+}$such that for every deformation functor $G$ and every natural transformation $\phi: F \rightarrow G$ there exists unique a natural transformation $\psi: F^{+} \rightarrow G$ such that $\phi=\psi \eta$. 
Proof We first define a functorial relation $\sim$ on the sets $F(A), A \in \mathbf{C}$; we set $a \sim b$ if and only if there exists $\epsilon>0$ and $x \in F\left(A[t, d t]_{\epsilon}\right)$ such that $e_{0}(x)=a, e_{1}(x)=b$. By 2.6 if $F$ is a deformation functor then $a \sim b$ if and only if $a=b$. Therefore if we define $F^{+}$as the quotient of $F$ by the equivalence relation generated by $\sim$ and $\eta$ as the natural projection, then there exists a unique $\psi$ as in the statement of the theorem. We only need to prove that $F^{+}$is a deformation functor.

SteP 1: If $C \in \mathbf{D G} \cap \mathbf{C}$ is acyclic then $F^{+}(C)=\{0\}$.

Since $C$ is acyclic there exists a homotopy $H: C \rightarrow C[t, d t]_{\epsilon}, \epsilon \leq 1$, such that $H_{0}=0$, $H_{1}=I d$; it is then clear that for every $x \in F(C)$ we have $x=H_{1}(x) \sim H_{0}(x)=0$.

STEP 2: $\sim$ is an equivalence relation on $F(A)$ for every $A \in \mathbf{C}$.

This is essentially standard (see e.g. [12, p. 125]). In view of the inclusion $A \rightarrow A[t, d t]_{\epsilon}$ the relation $\sim$ is reflexive. The symmetry is proved simply by remarking that the automorphism of dg-algebras

$$
A[t, d t] \rightarrow A[t, d t] ; \quad a \otimes p(t, d t) \rightarrow a \otimes p(1-t,-d t)
$$

preserves the subalgebras $A[t, d t]_{\epsilon}$ for every $\epsilon>0$.

Consider now $\epsilon>0$ and $x \in F\left(A[t, d t]_{\epsilon}\right), y \in F\left(A[s, d s]_{\epsilon}\right)$ such that $e_{0}(x)=e_{0}(y)$; we need to prove that $e_{1}(x) \sim e_{1}(y)$.

Write $\mathbb{K}[t, s, d t, d s]=\oplus_{n \geq 0} S^{n}$, where $S^{n}$ is the $n$-th symmetric power of the acyclic complex $\mathbb{K} t \oplus \mathbb{K} s \stackrel{d}{\longrightarrow} \mathbb{K} d t \oplus \mathbb{K} d s$ and define $A[t, s, d t, d s]_{\epsilon}=A \oplus \oplus_{n>0} A^{\lceil n \epsilon]} \otimes S^{n}$. There exists a commutative diagram

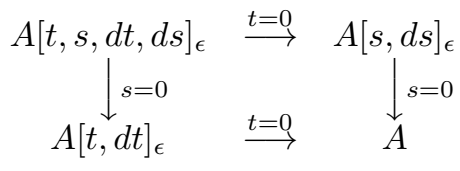

The kernel of the surjective morphism

$$
A[t, s, d t, d s]_{\epsilon} \stackrel{\eta}{\longrightarrow} A[t, d t]_{\epsilon} \times_{A} A[t, d t]_{\epsilon}
$$

is equal to $\oplus_{n>0} A^{\lceil n \epsilon\rceil} \otimes\left(S^{n} \cap I\right)$, where $I \subset \mathbb{K}[t, s, d t, d s]$ is the homogeneous differential ideal generated by $s t, s d t, t d s, d t d s$. Since $I \cap S^{n}$ is acyclic for every $n>0$, the morphism $\eta$ is a finite composition of acyclic small extensions.

Let $\xi \in F\left(A[t, s, d t, d s]_{\epsilon}\right)$ be a lifting of $(x, y)$ and let $z \in F\left(A[u, d u]_{\epsilon}\right)$ be the image of $\xi$ under the morphism

$$
A[t, s, d t, d s]_{\epsilon} \rightarrow A[u, d u]_{\epsilon}, \quad t \rightarrow 1-u, \quad s \rightarrow u
$$

The evaluation of $z$ gives $e_{0}(z)=e_{1}(x), e_{1}(z)=e_{1}(y)$.

Step 3: If $\alpha: A \rightarrow B$ is surjective then

$$
F\left(A[t, d t]_{\epsilon}\right) \stackrel{\left(e_{0}, \alpha\right)}{\longrightarrow} F(A) \times_{F(B)} F\left(B[t, d t]_{\epsilon}\right)
$$

is surjective.

It is not restrictive to assume $\alpha$ a small extension with kernel $I$. The kernel of $\left(e_{0}, \alpha\right)$ is equal to $\oplus_{n>0}\left(A^{\lceil n \epsilon\rceil} \cap I\right) \otimes\left(\mathbb{K} t^{n} \oplus \mathbb{K} t^{n-1} d t\right)$ and therefore $\left(e_{0}, \alpha\right)$ is an acyclic small extension. 
STEP 4: The functor $F^{+}$satisfies $2 \mathrm{a}$ of 2.1 .

Let $a \in F(A), b \in F(B)$ be such that $\alpha(a) \sim \beta(b)$; by Step 3 there exists $a^{\prime} \sim a, a^{\prime} \in F(A)$ such that $\alpha\left(a^{\prime}\right)=\beta(b)$ and then the pair $\left(a^{\prime}, b\right)$ lifts to $F\left(A \times_{C} B\right)$.

STEP 5: The functor $F^{+}$satisfies $2 \mathrm{~b}$ of 2.1.

By 2.2 it is sufficient to verify the condition separately for the cases $C=0$ and $B=0$. When $C=0$ the situation is easy: in fact $(A \times B)[t, d t]_{\epsilon}=A[t, d t]_{\epsilon} \times B[t, d t]_{\epsilon}, F((A \times$ $\left.B)[t, d t]_{\epsilon}\right)=F\left(A[t, d t]_{\epsilon}\right) \times F\left(B[t, d t]_{\epsilon}\right)$ and the relation $\sim$ over $F(A \times B)$ is the product of the relations $\sim$ over $F(A)$ and $F(B)$; this implies that $F^{+}(A \times B)=F^{+}(A) \times F^{+}(B)$.

Assume now $B=0$, then the fibred product $D:=A \times_{C} B$ is equal to the kernel of $\alpha$. We need to prove that the map $F^{+}(D) \rightarrow F^{+}(A)$ is injective. Let $a_{0}, a_{1} \in F(D) \subset F(A)$ and let $x \in F\left(A[t, d t]_{\epsilon}\right)$ be an element such that $e_{i}(x)=a_{i}, i=0,1$. Denote by $\bar{x} \in F\left(C[t, d t]_{\epsilon}\right)$ the image of $x$ by $\alpha$.

Since $C$ is acyclic there exists a morphism of graded vector spaces $\sigma: C \rightarrow C[-1]$ such that $d \sigma+\sigma d=I d$ and we can define a morphism of complexes

$$
h: C \rightarrow(\mathbb{K} s \oplus \mathbb{K} d s) \otimes C \subset C[s, d s]_{1} ; \quad h(v)=s \otimes v+d s \otimes \sigma(v)
$$

The morphism $h$ extends in a natural way to a morphism

$$
h: C[t, d t]_{\epsilon} \rightarrow(\mathbb{K} s \oplus \mathbb{K} d s) \otimes C[t, d t]_{\epsilon}
$$

such that for every scalar $\zeta \in \mathbb{K}$ there exists a commutative diagram

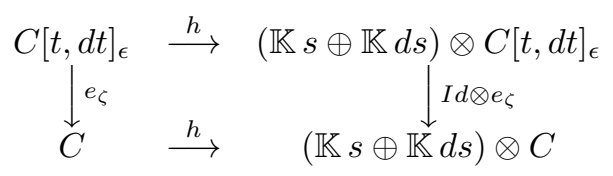

Setting $\bar{z}=h(\bar{x})$ we have $\bar{z}_{\mid s=1}=\bar{x}, \bar{z}_{\mid s=0}=\bar{z}_{\mid t=0}=\bar{z}_{\mid t=1}=0$. By step $3 \bar{z}$ lifts to an element $z \in F\left(A[t, d t]_{\epsilon}[s, d s]_{1}\right)$ such that $z_{\mid s=1}=x$; Now the specializations $z_{\mid s=0}, z_{\mid t=0}, z_{\mid t=1}$ are annihilated by $\alpha$ and therefore give a chain of equivalences in $F(D)$

$$
a_{0}=z_{\mid s=1, t=0} \sim z_{\mid s=0, t=0} \sim z_{\mid s=0, t=1} \sim z_{\mid s=1, t=1}=a_{1}
$$

proving that $a_{0} \sim a_{1}$ inside $F(D)$.

The combination of Steps 1,4 and 5 tell us that $F^{+}$is a deformation functor.

For later use we point out that in the previous proof (Steps 2,3) we have also showed the following

Lemma 2.9. Let $F: \mathbf{C} \rightarrow$ Set be a predeformation functor and let $\nu: F \rightarrow F^{+}$be the projection onto the associated deformation functor.

Then for every surjective morphism $A \rightarrow B$ in $\mathbf{C}$, the map

$$
F(A) \rightarrow F(B) \times_{F^{+}(B)} F^{+}(A)
$$

is surjective.

For computational purposes it is often useful the following

Lemma 2.10. Let $S$ be a complex of vector spaces and assume that the functor

$$
\mathbf{D G} \cap \mathbf{C} \rightarrow \mathbf{S e t} ; C \rightarrow Z^{1}(S \otimes C)
$$

is the restriction of a predeformation functor $F$. Then for every complex $C \in \mathbf{D G} \cap \mathbf{C}$ we have $F^{+}(C)=H^{1}(S \otimes C)$; in particular $T^{i} F^{+}=H^{i}(S)$. 
Proof Let $C \in \mathbf{D G} \cap \mathbf{C}$ be a fixed complex and set $A=S \otimes C$; since $A^{2}=C^{2}=0$ for every $\epsilon>0$ we have $A[t, d t]_{\epsilon}=S \otimes C[t, d t]_{\epsilon}$.

For every integer $i \geq 2$ let $p_{i}(t) \in \mathbb{K}[t]$ be a fixed monic polynomial of degree $i$ such that $p(0)=p(1)=0$; every element $\xi \in Z^{1}\left(A[t, d t]_{\epsilon}\right)=F\left(C[t, d t]_{\epsilon}\right)$ can be written as

$$
\xi=a_{0}+a_{1} t+\sum_{i \geq 2} a_{i} p_{i}(t)+b_{1} d t+\sum_{i \geq 2} b_{i} p_{i}^{\prime}(t) d t
$$

with $a_{i} \in A_{1}, b_{i} \in A_{0}, d a_{0}=0$ and $d b_{i}=a_{i}$ for every $i$. In particular $e_{1}(\xi)-e_{0}(\xi)=$ $a_{1}=d b_{1} \in B^{1}(A)$. Conversely if $a_{0}, a_{1} \in Z^{1}(A)$ and $a_{1}-a_{0}=d b_{1}$ for some $b_{1} \in A_{0}$ then $a_{j}=e_{j}\left(a_{0}+\left(a_{1}-a_{0}\right) t+b_{1} d t\right)$ for $j=0,1$.

Example 2.11. Let $R$ be an algebraically free complete differential algebra;

1. For every complex $C$ we have

$$
h_{R}(C)=\operatorname{Hom}_{\mathbf{D G}}\left(R / R^{2}, C\right)=Z^{0}\left(\operatorname{Hom}^{*}\left(R / R^{2}, \mathbb{K}\right) \otimes C\right)
$$

and then $T^{i} h_{R}^{+}=H^{i-1}\left(\operatorname{Hom}^{*}\left(R / R^{2}, \mathbb{K}\right)\right)$.

2. The functor $[R,-]:$ NDGA $\rightarrow$ Set is a deformation functor with tangent space

$$
T^{i}[R,-]=H^{i-1}\left(\operatorname{Hom}^{*}\left(R / R^{2}, \mathbb{K}\right)\right)=H^{i-1}\left(\left(R / R^{2}\right)^{\vee}\right) .
$$

The proof of this fact is essentially the same (but more easy) of 2.8 and it is omitted. If the graded vector space $R / R^{2}$ is finite-dimensional then it is easy to prove directly that $h_{R}^{+}=[R,-]$

3. The projection $h_{R} \rightarrow[R,-]$ factors through a morphism of deformation functors $h_{R}^{+} \rightarrow$ $[R,-]$ which is an isomorphism on tangent spaces.

All the deformation functors are quasismooth by definition; a stronger notion of smoothness is given by the natural generalization of the classical notion of smooth morphism.

Definition 2.12. A natural transformation of deformation functors $\theta: F \rightarrow G$ is called smooth if, for every morphism $A \rightarrow B$ in $\mathbf{C}$, which is surjective in homology the natural map

$$
F(A) \rightarrow F(B) \times_{G(B)} G(A)
$$

is surjective. A deformation functor $F$ is called smooth if the trivial morphism $F \rightarrow 0$ is smooth.

Proposition 2.13. Let $(S, d)$ be a minimal complete quasismooth differential algebra. Then the deformation functor $[S,-]$ is smooth if and only if $d=0$.

Proof If $d=0$ it is easy to see that $[S,-]$ is smooth. Conversely assume $d \neq 0$; let $n \geq 2$ be the greatest integer such that $d(S) \subset S^{n}$ and let $\pi: S / S^{2} \rightarrow V$ be a finite dimensional quotient such that the composition $H \stackrel{d}{\longrightarrow} S^{n} / S^{n+1} \rightarrow \odot^{n}(V)$ is nonzero.

Let $(R, 0)$ be the complete free differential algebra with $R / R^{2}=V$; we shall see that $\left[S, R / R^{n+1}\right] \rightarrow\left[S, R / R^{n}\right]$ is not surjective, this implies that $[S,-]$ is not smooth. Since $S / S^{n}$ and $R / R^{n}$ have differential $d=0$ we have $\left[S, R / R^{n}\right]=\operatorname{Hom}_{\mathbf{D G} \mathbf{A}}\left(S, R / R^{n}\right)$ and therefore it is sufficient to show that the projection $\pi: S \rightarrow R / R^{n}$ does not lift to $R / R^{n+1}$. Assume that there exists $f: S \rightarrow R / R^{n+1}$ which is a lifting of $\pi$; let $x \in S / S^{2}$ be such that $\pi(d x) \neq 0$ in $R^{n}$, then $f(x)=\pi(x)+\alpha$ for some $\alpha \in R^{n} / R^{n+1}$; in particular the restriction of $f$ to $S^{2}$ is equal to $\pi$. We must therefore have $0=d f(x)=f(d x)=\pi(d x) \neq 0$ giving a contradiction.

The second basic example of deformation functor is the deformation functor of a differential graded Lie algebra; we recall the well known 
Definition 2.14. A differential graded Lie algebra (DGLA in short terms) is a triple $(L, d,[]$, with $(L, d) \in \mathbf{D G}$ and $[]:, L \times L \rightarrow L$ is a bilinear map such that:

- $\left[L^{i}, L^{j}\right] \subset L^{i+j}$.

- $[x, y]+(-1)^{\bar{x}} \bar{y}[y, x]=0$.

- $\left(\right.$ Jacoby identity) $[x,[y, z]]=[[x, y], z]+(-1)^{\bar{x}} \bar{y}[y,[x, z]]$

- $d[x, y]=[d x, y]+(-1)^{\bar{x}}[x, d y]$

Example 2.15. Let $A$ be a dg-algebra, then the $A$-module $\operatorname{Der}^{*}(A, A)$ has a natural structure of DGLA with bracket $[\delta, \tau]=\delta \circ \tau-(-1)^{\bar{\delta}} \tau \circ \delta$, where the composition $\circ$ is made by considering $\delta$ and $\tau$ as linear endomorphism of the $\mathbb{K}$-vector space $A$.

We shall denote by DGLA the category of differential graded Lie algebras: a morphism of DGLA is simply a morphism of complexes which commutes with brackets.

The Maurer-Cartan elements of a DGLA $L$ are by definition

$$
M C(L)=\left\{x \in L^{1} \mid d x+\frac{1}{2}[x, x]=0\right\}
$$

Clearly every morphism of differential graded Lie algebras $L \rightarrow N$ sends the Maurer-Cartan elements of $L$ into the ones of $N$.

Given a DGLA $L$ and $A \in \mathbf{C}$, the tensor product $L \otimes A$ has a natural structure of nilpotent DGLA with

$$
\begin{gathered}
(L \otimes A)^{i}=\oplus_{j \in \mathbb{Z}} L^{j} \otimes A_{i-j} \\
d(x \otimes a)=d x \otimes a+(-1)^{\bar{x}} x \otimes d a \\
{[x \otimes a, y \otimes b]=(-1)^{\bar{a} \bar{y}}[x, y] \otimes a b}
\end{gathered}
$$

Every morphism of DGLA, $L \rightarrow N$ and every morphism $A \rightarrow B$ in $\mathbf{C}$ give a natural commutative diagram of morphisms of differential graded Lie algebras

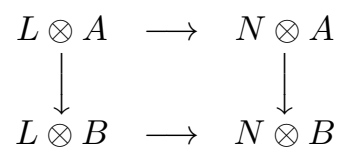

Definition 2.16. The Maurer-Cartan functor $M C_{L}: \mathbf{C} \rightarrow \mathbf{S e t}$ of a DGLA L is given by

$$
M C_{L}(A)=M C(L \otimes A)
$$

Lemma 2.17. In the notation above $M C_{L}$ is a predeformation functor; the resulting functor $M C:$ DGLA $\rightarrow$ PreDef is faithful. 
Proof It is evident that $M C_{L}(0)=0$ and for every pair of morphisms $\alpha: A \rightarrow C, \beta: B \rightarrow C$ in $\mathbf{C}$ we have

$$
M C_{L}\left(A \times_{C} B\right)=M C_{L}(A) \times_{M C_{L}(C)} M C_{L}(B)
$$

Let $0 \longrightarrow I \longrightarrow A \longrightarrow B \longrightarrow 0$ be an acyclic small extension and $x \in M C_{L}(B)$. Since $\alpha$ is surjective there exists $y \in(L \otimes A)^{1}$ such that $\alpha(y)=x$. Setting

$$
h=d y+\frac{1}{2}[y, y] \in(L \otimes I)^{2}
$$

we have

$$
d h=\frac{1}{2} d[y, y]=[d y, y]=[h, y]-\frac{1}{2}[[y, y], y] .
$$

By Jacoby identity $[[y, y], y]=0$ and, since $A I=0$ also $[h, y]=0$; thus $d h=0$ and, being $L \otimes I$ acyclic by Künneth formula, there exists $s \in(L \otimes I)^{1}$ such that $d s=h$. The element $y-s$ lifts $x$ and satisfies Maurer-Cartan equation. We have thus proved that $M C_{L}$ is a predeformation functor.

The natural identification $L^{i}=M C_{L}((\mathbb{K} t \oplus \mathbb{K} d t)[i])$ shows that $M C:$ DGLA $\rightarrow$ PreDef is faithful.

Remark 2.18. It is easy to prove that a differential graded Lie algebra can be recovered, up to isomorphism, from its Maurer-Cartan functor.

In order to motivate our definition of deformation functor we point out that, if $A \rightarrow B$ is a surjective quasiisomorphism in $\mathbf{C}$, then in general $M C_{L}(A) \rightarrow M C_{L}(B)$ is not surjective. As an example take $\mathbb{K}$ algebraically closed and $L$ a finite-dimensional non-nilpotent Lie algebra, considered as a DGLA concentrated in degree 0. Fix $a \in L$ such that $a d(a): L \rightarrow L$ has an eigenvalue $\lambda \neq 0$. Up to multiplication of $a$ by $-\lambda^{-1}$ we can assume $\lambda=-1$. Let $V \subset L$ be the image of $a d(a)$, the linear map $I d+a d(a): V \rightarrow V$ is not surjective and then there exists $b \in L$ such that the equation $x+[a, x]+[a, b]=0$ has no solution in $L$.

Let $u, v, w$ be indeterminates of degree 1 and consider the dg-algebras

$$
\begin{gathered}
B=\mathbb{K} u \oplus \mathbb{K} v, \quad B^{2}=0, d=0 \\
A=\mathbb{K} u \oplus \mathbb{K} v \oplus \mathbb{K} w \oplus \mathbb{K} d w, \quad u v=u w=d w, v w=0
\end{gathered}
$$

The projection $A \rightarrow B$ is a quasiisomorphism but the element $a \otimes u+b \otimes v \in M C_{L}(B)$ cannot lifted to $M C_{L}(A)$. In fact, if there exists $\xi=a \otimes u+b \otimes v+x \otimes w \in M C_{L}(A)$, then

$$
0=d \xi+\frac{1}{2}[\xi, \xi]=(x+[a, x]+[a, b]) \otimes d w
$$

in contradiction with the previous choice of $a, b$.

To every differential graded Lie algebra $(L, d,[]$,$) we can associate a new DGLA \left(L_{d}, d_{d},[,]_{d}\right)$ by setting $L_{d}=L \oplus \mathbb{K} d$, where $d$ is considered as an indeterminate of degree $1, d_{d}(a+\alpha d)=$ $d(a)$ and

$$
[a+\alpha d, b+\beta d]_{d}=[a, b]+\alpha d(b)-(-1)^{\bar{a}} \beta d(a)
$$

Note that $a \in M C(L)$ if and only if $a \in L^{1}$ and $[a+d, a+d]_{d}=0$.

If $L^{0}=L_{d}^{0}$ is a nilpotent Lie algebra then the adjoint action $L^{0} \times L_{d}^{1} \stackrel{[,]}{\longrightarrow} L^{1} \subset L_{d}^{1}$ gives a group action $\exp \left(L^{0}\right) \times L_{d}^{1} \rightarrow L_{d}^{1}$ preserving the affine hyperplane $L^{1}+\alpha d$ for every fixed $\alpha \in \mathbb{K}$. As a consequence of Jacoby identity (see [2], [8] for details) this action preserves 
the quadratic cone $\left\{x \in L_{d}^{1} \mid[x, x]_{d}=0\right\}$ and then $\exp \left(L^{0}\right)$ acts, via the above identification, on the set $M C(L)$.

The restriction of the adjoint action to $M C(L)$ is usually called "gauge action". Note that if, for $a \in L^{0}$ and $b \in M C(L)$, we have $[a, b+d]_{d}=[a, b]-d a=0$ then $e^{a} b=b$; in particular th set $K_{b}=\left\{[b, c]+d c \mid c \in L^{-1}\right\}$ is a Lie subalgebra of $L^{0}$ and $\exp \left(K_{b}\right)$ is contained in the stabilizer of $b$.

For every DGLA $L$ and every $A \in \mathbf{C}$ we define $\operatorname{Def}_{L}(A)$ as the quotient of $M C(L \otimes A)$ by the gauge action of the group $\exp \left((L \otimes A)^{0}\right)$. The gauge action commutes with morphisms in $\mathbf{C}$ and with morphisms of differential graded Lie algebras and then the above definition gives a functor $\operatorname{Def}_{L}: \mathbf{C} \rightarrow$ Set called the deformation functors of $L$ : in fact we have

Theorem 2.19. For every differential graded Lie algebra $L, \operatorname{Def}_{L}$ is a deformation functor with $T^{i} \operatorname{Def}_{L}=H^{i}(L)$.

Proof If $C \in \mathbf{D G} \cap \mathbf{C}$ then $L \otimes C$ is an abelian DGLA, $M C_{L}(C)=Z^{1}(L \otimes C)$ and the gauge action is given by $e^{a} b=b-d a, b \in(L \otimes C)^{1}, a \in(L \otimes C)^{0}$. This proves that $\operatorname{Def}_{L}(C)=H^{1}(L \otimes C)$ and in particular $T^{i} \operatorname{Def}_{L}=H^{1}(L \otimes \mathbb{K}[i-1])=H^{i}(L)$.

Since $\operatorname{Def}_{L}$ is the quotient of a predeformation functor and $\operatorname{Def}_{L}(I)=0$ for every acyclic $I \in \mathbf{D G} \cap \mathbf{C}$, it is sufficient to verify the generalized Schlessinger's conditions. Let $\alpha: A \rightarrow$ $C, \beta: B \rightarrow C$ morphism in $\mathbf{C}$ with $\alpha$ surjective. Assume there are given $a \in M C_{L}(A)$, $b \in M C_{L}(B)$ such that $\alpha(a)$ and $\beta(b)$ give the same element in $\operatorname{Def}_{L}(C)$; then there exists $u \in(L \otimes C)^{0}$ such that $\beta(b)=e^{u} \alpha(a)$. Let $v \in(L \otimes A)^{0}$ be a lifting of $u$, changing if necessary $a$ with its gauge equivalent element $e^{v} a$, we may suppose $\alpha(a)=\beta(b)$, the pair $(a, b)$ lifts to $M C_{L}\left(A \times_{C} B\right)$; this proves that the map

$$
\operatorname{Def}_{L}\left(A \times_{C} B\right) \rightarrow \operatorname{Def}_{L}(A) \times \operatorname{Def}_{L}(C) \operatorname{Def}_{L}(B)
$$

is surjective.

If $C=0$ then the gauge action $\exp \left((L \otimes(A \times B))^{0}\right) \times M C_{L}(A \times B) \rightarrow M C_{L}(A \times B)$ is the direct product of the gauge actions $\exp \left((L \otimes A)^{0}\right) \times M C_{L}(A) \rightarrow M C_{L}(A)$ and $\exp ((L \otimes$ $\left.B)^{0}\right) \times M C_{L}(B) \rightarrow M C_{L}(B)$ and therefore $\operatorname{Def}_{L}(A \times B)=\operatorname{Def}_{L}(A) \times \operatorname{Def}_{L}(B)$.

Finally assume $B=0, C$ acyclic with $C^{2}=0$ and set $D=\operatorname{ker} \alpha \simeq A \times_{C} B$. Let $a_{1}, a_{2} \in M C_{L}(D), u \in(L \otimes A)^{0}$ be such that $a_{2}=e^{u} a_{1}$; we need to prove that there exists $v \in(L \otimes D)^{0}$ such that $a_{2}=e^{v} a_{1}$.

Since $\alpha\left(a_{1}\right)=\alpha\left(a_{2}\right)=0$ and $L \otimes C$ is an abelian DGLA we have $0=e^{\alpha(u)} 0=0-d \alpha(u)$ and then there exists $h \in(L \otimes A)^{-1}$ such that $d \alpha(h)=-\alpha(u), u+d h \in(L \otimes D)^{0}$. If $w=\left[a_{1}, h\right]+d h$ then $e^{w} a_{1}=a_{1}, e^{u} e^{w} a_{1}=e^{v} a_{1}=a_{2}$ where $v=u * w$ is determined by Baker-Campbell-Hausdorff formula.

We claim that $v \in L \otimes D$; in fact $v=u * w \equiv u+w \equiv u+d h(\bmod [L \otimes A, L \otimes A])$ and since $A^{2} \subset D$ we have $v=u * w \equiv u+d h \equiv 0(\bmod L \otimes D)$.

Corollary 2.20. For every differential graded Lie algebra $L$ the natural projection $M C_{L} \rightarrow$ Def $_{L}$ induces (by 2.8) a morphism $M C_{L}^{+} \rightarrow \operatorname{Def}_{L}$ which is an isomorphism on tangent spaces.

\section{Obstruction theory and the inverse function theorem for deformation functors}

Theorem 3.1. A morphism of predeformation functors $\theta: F \rightarrow G$ is an isomorphism if and only if $\theta: F(A) \rightarrow G(A)$ is a bijection for every complex $A \in \mathbf{C} \cap \mathbf{D G}$. 
Proof The proof uses the natural generalization to the differential graded case of some standard techniques in Schlessinger's theory, cf. [6].

Let $\theta: F \rightarrow G$ be a fixed natural transformation of predeformation functors such that $\theta: F(A) \rightarrow G(A)$ is bijective for every $A \in \mathbf{D G} \cap \mathbf{C}$.

SteP 1: For every small extension

$$
0 \longrightarrow I \longrightarrow A \stackrel{\alpha}{\longrightarrow} B \longrightarrow 0
$$

and every $b \in F(B)$ we have either $\alpha^{-1}(b)=\emptyset$ or $\theta\left(\alpha^{-1}(b)\right)=\alpha^{-1}(\theta(b))$.

As in the classical case [23], there exists an isomorphism of dg-algebras

$$
A \times I \longrightarrow A \times{ }_{B} A ; \quad(a, t) \rightarrow(a, a+t)
$$

and then for every predeformation functor $E$ there exists a natural surjective map

$$
\vartheta_{E}: E(A) \times E(I)=E(A \times I) \rightarrow E(A) \times_{E(B)} E(A)
$$

This implies in particular that there exists a natural transitive action of the vector space $E(I)$ on the fibres of the map $E(A) \rightarrow E(B)$. Moreover this action commutes with natural transformations of functors.

In our case we have a commutative diagram

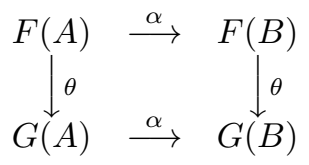

and compatible transitive actions of the vector space $F(I)=G(I)$ on the fibres of the horizontal maps. This proves Step 1.

Step 2: Let $A \stackrel{\alpha}{\longrightarrow} V$ be a surjective morphism in $\mathbf{C}$ with $V \in \mathbf{D G} \cap \mathbf{C}$ acyclic. Denoting by $\iota: B=\operatorname{ker} \alpha \rightarrow A$ the inclusion, then an element $b \in G(B)$ lifts to $F(B)$ if and only if $\iota(b)$ lifts to $F(A)$.

In fact, by condition $2 b$ of 2.1, the inclusion $\iota$ gives bijection $F(B)=\alpha^{-1}(0) \subset F(A)$, $G(B)=\alpha^{-1}(0) \subset G(A)$. Since $F(V)=G(V)$ we have

$$
\iota \theta(F(B))=\theta(F(A)) \cap \alpha^{-1}(0)=\theta(F(A)) \cap \iota(G(B))
$$

STEP 3: Let

$$
0 \longrightarrow I \stackrel{\iota}{\longrightarrow} A \stackrel{\alpha}{\longrightarrow} B \longrightarrow 0
$$

be a small extension and $b \in F(B)$. Then $b$ lifts to $F(A)$ if and only if $\theta(b)$ lifts to $G(A)$.

The only if part is trivial, let's prove the if part. Let $\tilde{a} \in G(A)$ be a lifting of $\theta(b)$ and let $C=A \oplus I[1]$ be the mapping cone of $\iota$. The projection $\pi: C \rightarrow B$ is a small acyclic extension and then there exists $b_{1} \in F(C)$ which lifts $b$. By Step 1 we can assume that $\theta\left(b_{1}\right)=j(\tilde{a})$, where $j: A \rightarrow C$ is the inclusion.

If $o b: C \rightarrow I[1]$ denotes the projection then $o b\left(\theta\left(b_{1}\right)\right)=0$ and since $F(I[1])=G(I[1])$ we also have $o b\left(b_{1}\right)=0$; by generalized Schlessinger's conditions $b_{1}$ lifts to $F(A)$. 
Step 4: For every $A \in \mathbf{C}$ the map $\theta: F(A) \rightarrow G(A)$ is surjective.

By induction over $\operatorname{dim}_{\mathbb{K}} A$ we can assume that $F(B)$ goes surjectively onto $G(B)$ for every proper quotient $B=A / I$. Let

$$
0 \longrightarrow I \longrightarrow A \stackrel{\alpha}{\longrightarrow} B \longrightarrow 0
$$

be a small extension with $I \neq 0, \tilde{a} \in G(A)$ a fixed element and $b \in F(B)$ such that $\theta(b)=$ $\alpha(\tilde{a})$. By Step $3 \alpha^{-1}(b)$ is not empty and then by Step $1 \tilde{a} \in \theta(F(A))$.

SteP 5: Let $a \in F(A)$, for every surjective morphism $f: A \rightarrow B$ in the category $\mathbf{C}$ we define

$$
S_{F}(a, f)=\left\{\xi \in F\left(A \times_{B} A\right) \mid \xi \rightarrow(a, a) \in F(A) \times_{F(B)} F(A)\right\}
$$

By definition, if $f$ is a small extension and $I=\operatorname{ker} f$ then $S_{F}(a, f)$ is naturally isomorphic to the stabilizer of $a$ under the action of $F(I)$ on the fibre $f^{-1}(f(a))$. It is also clear that $\theta\left(S_{F}(a, f)\right) \subset S_{G}(\theta(a), f)$.

STEP 6: For every $a \in F(A)$ and every surjective morphism $f: A \rightarrow B$ the map $\theta: S_{F}(a, f) \rightarrow S_{G}(\theta(a), f)$ is surjective.

This is trivially true if $B=0$, we prove the general assertion by induction on $\operatorname{dim}_{\mathbb{K}} B$. Let

$$
0 \longrightarrow I \longrightarrow B \stackrel{\alpha}{\longrightarrow} C \longrightarrow 0
$$

be a small extension with $I \neq 0$, set $g=\alpha f$ and denote by $h: A \times_{C} A \rightarrow I$ the surjective morphism of dg-algebras defined by $h\left(a_{1}, a_{2}\right)=f\left(a_{1}\right)-f\left(a_{2}\right)$; the kernel of $h$ is $A \times_{B} A$, let's denote by $\iota: A \times_{B} A \rightarrow A \times_{C} A$ the natural inclusion.

By generalized Schlessinger's conditions the maps

$$
F\left(A \times_{B} A\right) \rightarrow \operatorname{ker}\left(F\left(A \times_{C} A\right) \stackrel{h}{\longrightarrow} F(I)\right) ; \quad S_{F}(a, f) \rightarrow S_{F}(a, g) \cap \operatorname{ker} h
$$

are surjective. Let $\tilde{\xi} \in S_{G}(\theta(a), f)$ and let $\eta \in S_{F}(a, g)$ such that $\theta(\eta)=\iota(\tilde{\xi})$. Since $F(I)=$ $G(I)$ we have $h(\eta)=0$ and then $\eta$ lifts to some $\xi_{1} \in S_{F}(a, f)$. Let $D=\left(A \times_{C} A\right) \oplus I[-1]$ be the derived inverse mapping cone of $h$, it is immediate to check that the projection maps

$$
\pi_{1}: D \rightarrow A \times_{C} A, \quad\left(h, \pi_{2}\right): D \rightarrow I \oplus I[-1]
$$

are surjective morphisms of dg-algebras, $I \oplus I[-1]$ is an acyclic complex and the kernel of $\left(h, \pi_{2}\right)$ is exactly $A \times_{B} A$; again by generalized Schlessinger's conditions $F\left(A \times_{B} A\right) \subset F(D)$, $G\left(A \times_{B} A\right) \subset G(D)$. Moreover there exists a cartesian diagram

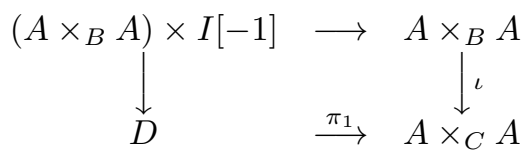

which gives surjective maps

$$
\begin{aligned}
& F\left(A \times_{B} A\right) \times F(I[-1]) \stackrel{\varrho}{\longrightarrow} F\left(A \times_{B} A\right) \times_{F\left(A \times_{C} A\right)} F(D) \\
& G\left(A \times_{B} A\right) \times G(I[-1]) \stackrel{\varrho}{\longrightarrow} G\left(A \times_{B} A\right) \times_{G\left(A \times_{C} A\right)} G(D)
\end{aligned}
$$

This implies the existence of $v \in G(I[-1])=F(I[-1])$ such that $\varrho\left(\theta\left(\xi_{1}\right), v\right)=\left(\theta\left(\xi_{1}\right), \tilde{\xi}\right)$; defining $\xi \in F(D)$ by the formula $\varrho\left(\xi_{1}, v\right)=\left(\xi_{1}, \xi\right)$ we get $\theta(\xi)=\tilde{\xi}$ and then, by Step 2, $\xi \in S_{F}(a, f)$. 
Step 7: For every $A \in \mathbf{C}$ the map $\theta: F(A) \rightarrow G(A)$ is injective.

This is true by assumption if $A^{2}=0$; if $A^{2} \neq 0$ we can suppose by induction that there exists a small extension

$$
0 \longrightarrow I \stackrel{\iota}{\longrightarrow} A \stackrel{\alpha}{\longrightarrow} B \longrightarrow 0
$$

with $I \neq 0$ and $\theta: F(B) \rightarrow G(B)$ injective.

Let $a_{1}, a_{2} \in F(A)$ be two elements such that $\theta\left(a_{1}\right)=\theta\left(a_{2}\right)$; by assumption $f\left(a_{1}\right)=f\left(a_{2}\right)$ and then there exists $t \in F(I)$ such that $\vartheta_{F}\left(a_{1}, t\right)=\left(a_{1}, a_{2}\right), \vartheta_{G}\left(\theta\left(a_{1}\right), \theta(t)\right)=\left(\theta\left(a_{1}\right), \theta\left(a_{2}\right)\right)$ and then $\theta(t) \in S_{G}\left(\theta\left(a_{1}\right), \alpha\right)$. By Step 6 there exists $s \in S_{F}\left(a_{1}, \alpha\right)$ such that $\theta(s)=\theta(t)$ and by injectivity of $\theta: F(I) \rightarrow G(I)$ we get $s=t$ and then $a_{1}=a_{2}$.

The result of Theorem 3.1 is particularly useful for morphisms of deformation functors: In fact we have

Lemma 3.2. Let $F: \mathbf{C} \rightarrow$ Set be a deformation functor; for every $I \in \mathbf{D G} \cap \mathbf{C}$ there exists a natural isomorphism

$$
F(I)=\underset{i \in \mathbb{Z}}{\oplus} T F[1]^{i} \otimes H_{-i}(I)=\underset{i \in \mathbb{Z}}{\oplus} T^{i+1} F \otimes H_{-i}(I)=H^{0}\left(\operatorname{Hom}^{*}\left(I^{\vee}, T F[1]\right)\right) .
$$

Proof Let $s: H_{*}(I) \rightarrow Z_{*}(I)$ be a linear section of the natural projection, then the composition of $s$ with the natural embedding $Z_{*}(I) \stackrel{\iota}{\longrightarrow} I$ is unique up to homotopy and its cokernel is an acyclic complex, therefore it gives a well defined isomorphism $F\left(H_{*}(I)\right) \rightarrow F(I)$. This says that it is not restrictive to prove the lemma for complexes with zero differential. Moreover since $F$ commutes with direct sum of complexes we can reduce to consider the case when $I \simeq \mathbb{K}^{s}[n]$ is a vector space concentrated in degree $-n$. Every $v \in I$ gives a morphism $F(\mathbb{K}[n]) \stackrel{v}{\longrightarrow} F(I)$ and we can define a natural map $T^{1+n} F \otimes I \rightarrow F(I), x \otimes v \rightarrow v(x)$. It is easy to verify that this map is an isomorphism of vector spaces.

As an immediate consequence we have:

Corollary 3.3. A morphism of deformation functors $\theta: F \rightarrow G$ is an isomorphism if and only if it gives an isomorphism of tangent spaces $\theta: T F[1] \stackrel{\simeq}{\longrightarrow} T G[1]$

Corollary 3.4. For every differential graded Lie algebra $L$ there exists a natural isomorphism $M C_{L}^{+}=\operatorname{Def}_{L}$.

Proof According to 2.8 the projection $M C_{L} \rightarrow$ Def $_{L}$ induces a natural transformation $M C_{L}^{+} \rightarrow \operatorname{Def}_{L}$ which is an isomorphism on tangent spaces by 2.20 .

Corollary 3.5. Every quasiisomorphism $\phi: L \rightarrow N$ of differential graded Lie algebras induces an isomorphism $\operatorname{Def}_{L} \simeq \operatorname{Def}_{N}$ between deformation functors.

Proof Trivial consequence of 2.19 and 3.3 .

Corollary 3.6. For every complete quasismooth $d g$-algebra $R$ there exists an isomorphism $h_{R}^{+}=[R,-]$. 
Proof Follows from 2.11 and 3.3. Note that this is a nontrivial result when the graded vector space $R / R^{2}$ is infinite dimensional.

The argument used in the proof of 3.1 can be used to show the existence of a complete natural (and probably universal, see [6] for the definition) obstruction theory for every deformation functor $F$.

Given a small extension

$$
e: \quad 0 \longrightarrow I \stackrel{\iota}{\longrightarrow} A \stackrel{\alpha}{\longrightarrow} B \longrightarrow 0
$$

we can define an "obstruction map" $o b_{e}: F(B) \rightarrow F(I[1])=H^{0}(T F[2] \otimes I)$ in the following way:

Let $C=A \oplus I[1]$ be the mapping cone of the inclusion $\iota$; since the projection $C \rightarrow B$ is an acyclic small extension, the projection $C \rightarrow I[1]$ gives a map $o b_{e}: F(B)=F(C) \rightarrow F(I[1])$.

The obstruction maps satisfy the following properties:

- $o b_{e}(b)=0$ if and only if $b$ lifts to $F(A)$.

- (naturality) The obstruction maps commute with natural transformation of functors.

- (base change) Given a morphism of small extensions

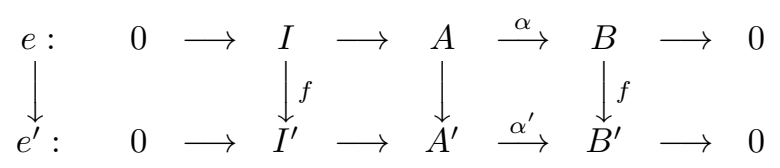

we have $f \circ o b_{e}=o b_{e^{\prime}} \circ f: F(B) \rightarrow F\left(I^{\prime}[1]\right)$.

The last two items are straightforward, while the first follows by generalized Schlessinger's conditions applied to the cartesian diagram

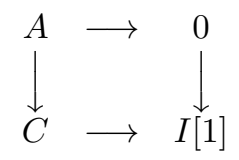

Example 3.7. If $0 \longrightarrow I \longrightarrow J \longrightarrow L \longrightarrow 0$ is a short exact sequence of complexes then the obstruction map $F(L) \rightarrow F(I[1])$ is the tensor product of the identity over $T F$ and the connecting homomorphism $H_{*}(L) \rightarrow H_{*}(I[1])$.

Remark 3.8. The above construction also shed light on the obstruction theory for general extensions. In this case it is necessary to replace obstruction maps with obstruction sections. If

$$
e: \quad 0 \longrightarrow I \stackrel{\iota}{\longrightarrow} A \stackrel{\alpha}{\longrightarrow} B \longrightarrow 0 ; \quad I^{2}=0
$$

is an extension, $C=A \oplus I[1]$ is the mapping cone of the inclusion and $B \oplus I[1]$ is the trivial extension (i.e. the mapping cone of the zero map $I[1] \stackrel{0}{\longrightarrow} B$ ) then the obstruction section $o b_{e}: F(B)=F(C) \rightarrow F(B \oplus I[1])$ is the map induced by the natural projection $C \rightarrow B \oplus I[1]$. The same proof as above shows that $b \in F(B)$ lifts to $F(A)$ if and only if ob $b_{e}(b)=j(b)$, where $j: F(B) \rightarrow F(B \oplus I[1])$ is the "zero section" induced by the natural inclusion $B \subset B \oplus I[1]$. Clearly if $e$ is a small extension then $B I=0, F(B \oplus I[1])=F(B) \times F(I[1])$ and we recover the notion of obstruction maps. 
Example 3.9. (Primary obstruction maps)

Let $u, v$ be indeterminates of degrees $-i,-j$ respectively and $F: \mathbf{C} \rightarrow$ Set a deformation functor; let $(u, v) \subset \mathbb{K}[u, v]$ be the ideal generated by $u, v$ and denote by

$$
Q_{i j}^{1}: T^{1+i} F \times T^{1+j} F=F(\mathbb{K} u \oplus K v) \rightarrow F(\mathbb{K} u v[1])=T^{2+i+j} F
$$

the obstruction map associated to the small extension

$$
0 \longrightarrow \mathbb{K} u v \longrightarrow \frac{(u, v)}{\left(u^{2}, v^{2}\right)} \longrightarrow \mathbb{K} u \oplus K v \longrightarrow 0 .
$$

In the realm of obstruction theory belong the following results which we will use in the next sections.

Consider a generic small extension in $\mathbf{C}$

$$
e: \quad 0 \longrightarrow I \stackrel{\iota}{\longrightarrow} A \stackrel{\alpha}{\longrightarrow} B \longrightarrow 0
$$

with obstruction map $o b_{e}: F(B) \rightarrow F(I[1])$. Let's denote by $d_{0}: A \rightarrow A[1]$ the differential of A.

For every morphism of dg-algebras $\phi: B \rightarrow I[1]$ the map $d_{\phi}=d_{0}+\iota \phi \alpha: A \rightarrow A[1]$ is a new differential on $A$ : in fact $B I=0$, this implies that $\iota \phi \alpha$ is a derivation and

$$
d_{\phi}^{2}=d_{0} \iota \phi \alpha+\iota \phi \alpha d_{0}=\iota\left(d_{I} \phi+\phi d_{B}\right) \alpha=\iota\left(-d_{I[1]} \phi+\phi d_{B}\right) \alpha=0 .
$$

Denote by $A_{\phi}$ the graded algebra $A$ with the differential $d_{\phi}$ and by

$$
e_{\phi}: \quad 0 \longrightarrow I \stackrel{\iota}{\longrightarrow} A_{\phi} \stackrel{\alpha}{\longrightarrow} B \longrightarrow 0
$$

the corresponding small extension.

Lemma 3.10. In the notation above $o b_{e_{\phi}}=o b_{e}+\phi: F(B) \rightarrow F(I[1])$.

Proof Let $A_{\phi} \oplus I[1], A \oplus I[1]$ be respectively the mapping cones of the inclusions $I \stackrel{\iota}{\longrightarrow} A_{\phi}$, $I \stackrel{\iota}{\longrightarrow} A$ and define the following isomorphism of graded algebras

$$
h=\left(\begin{array}{cc}
I d & 0 \\
\phi \alpha & I d
\end{array}\right): A_{\phi} \oplus I[1] \rightarrow A \oplus I[1]
$$

Since

$$
\left(\begin{array}{cc}
I d & 0 \\
\phi \alpha & I d
\end{array}\right)\left(\begin{array}{cc}
d_{\phi} & \iota \\
0 & d_{I[1]}
\end{array}\right)=\left(\begin{array}{cc}
d_{0} & \iota \\
0 & d_{I[1]}
\end{array}\right)\left(\begin{array}{cc}
I d & 0 \\
\phi \alpha & I d
\end{array}\right)
$$

the morphism $h$ is also an isomorphism of dg-algebras. There exists a commutative diagram

$$
\begin{array}{ccc}
A_{\phi} \oplus I[1] & \stackrel{h}{\longrightarrow} & A \oplus I[1] \\
\underset{\downarrow}{\downarrow} \alpha \times I d & & \downarrow \alpha \times I d \\
B \times I[1] & \stackrel{l}{\longrightarrow} & B \times I[1]
\end{array} \quad l=\left(\begin{array}{cc}
I d & 0 \\
\phi & I d
\end{array}\right)
$$

showing that, via the bijections $\alpha: F\left(A_{\phi} \oplus I[1]\right) \stackrel{\simeq}{\longrightarrow} F(B), \alpha: F(A \oplus I[1]) \stackrel{\simeq}{\longrightarrow} F(B)$ the isomorphism $h$ acts trivially on $F(B)$. Therefore the obstruction section $I d \times o b_{e_{\phi}}: F(B) \rightarrow$ $F(B) \times F(I[1])$ is precisely the composition with $l$ of the obstruction section $I d \times o b_{e}$.

Let's consider two dg-algebras $\left(B, d_{B}\right) \in \mathbf{C},\left(I, d_{I}\right) \in \mathbf{C} \cap \mathbf{D G}$ and a small extension of nilpotent graded algebras

$$
0 \longrightarrow I \stackrel{\iota}{\longrightarrow} A \stackrel{\alpha}{\longrightarrow} B \longrightarrow 0
$$

Assume that there exists a lifting of $d_{B}$ to a derivation $d \in \operatorname{Der}(A, A[1])$ such that $d_{\mid I}=d_{I}$. Such a lifting is not unique, any two liftings differ by an derivation of the form $\iota \phi \alpha$ for some derivation $\phi: B \rightarrow I[1]$. 
Lemma 3.11. In the above notation, the derivation $d^{2}=\frac{1}{2}[d, d]: A \rightarrow A[2]$ may be factored as $d^{2}=\delta \alpha$, where $\delta: B \rightarrow I[2]$ is a morphism of dg-algebras whose homotopy class $[\delta] \in$ $[B, I[2]]$ is independent from the choice of the lifting. Moreover $[\delta]=0$ if and only if there exists a lifting $d$ as above such that $d^{2}=0$.

Proof It is evident that $d^{2}(A) \subset I[2], d^{2}(I)=0$ and then $d^{2}$ induces a derivation $\delta: B \rightarrow$ $I[2]$; since $d^{2} \circ d=d_{I} \circ d^{2}=d_{I[2]} \circ d^{2}$ we have that $\delta$ is also a morphism of dg-algebras and then gives a morphism of complexes $\bar{\delta}: B / B^{2} \rightarrow I[2]$.

If $d^{\prime}$ is another lifting as above and $\phi=d^{\prime}-d$, then the derivation $\phi$ comes in the obvious way from a morphism of graded vector spaces $\bar{\phi}: B / B^{2} \rightarrow I[1]$; since $\delta^{\prime}-\delta=(d+\phi)^{2}-d^{2}=$ $d \phi+\phi d$ we have that $\bar{\phi}$ is the required homotopy between $\bar{\delta}$ and $\overline{\delta^{\prime}}$. If $\delta: B \rightarrow I[2]$ is homotopic to 0 then, being $I[2][t, d t]^{2}=0$, also $\bar{\delta}$ is homotopic to 0 and then there exists a derivation $\phi: A \rightarrow B \rightarrow B / B^{2} \rightarrow I[1]$ such that $d^{2}=d \phi+\phi d,(d-\phi)^{2}=0$.

In the above set-up, for every deformation functor $F$, the map $\delta: F(B) \rightarrow F(I[2])$ is, a priori, independent from the choice of the lifting of the differential. A posteriori we have the following stronger result:

Proposition 3.12. In the above notation $\delta(F(B))=0$; in particular if there exists $\xi \in F(B)$ such that the map

$$
[B, I[2]] \rightarrow F(I[2]) ; \quad[f] \rightarrow f(\xi)
$$

is injective then there exists a dg-algebra structure on $A$ making $\quad 0 \longrightarrow I \stackrel{\iota}{\longrightarrow} A \stackrel{\alpha}{\longrightarrow} B \longrightarrow 0$ a small extension in $\mathbf{C}$.

Proof We have already seen that $\delta$ induces a morphism of complexes $\bar{\delta}: B / B^{2} \rightarrow I[2]$. It is sufficient to construct a commutative diagram in $\mathbf{C}$

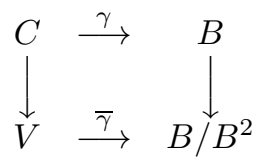

such that $\gamma$ is an acyclic small extension, $V^{2}=0$ and $\bar{\delta} \bar{\gamma}\left(H_{*}(V)\right)=0 \subset H_{*}(I[2])$.

Our solution is to define $C=A \times I[1]$ as a graded algebra and $\gamma$ as the composition of $\alpha$ with the projection on the first factor. Over $C$ we put the differential

$$
d_{C}=\left(\begin{array}{cc}
d & \iota \\
-d^{2} & d_{I[1]}
\end{array}\right): A \times I[1] \rightarrow A[1] \times I[2]
$$

A simple verification shows that $d_{C}$ is a derivation, $d_{C}^{2}=0$ and $\gamma$ is a morphism of dgalgebras. The kernel of $\gamma$ is the mapping cone of the identity $I \rightarrow I$ and therefore $\gamma$ is an acyclic small extension.

Finally we set $V=B / B^{2} \times I[1]$ and the differential $d_{V}$ is forced by $d_{C}$ to be equal to

$$
d_{V}=\left(\begin{array}{cc}
d_{B / B^{2}} & 0 \\
-\bar{\delta} & d_{I[1]}
\end{array}\right)
$$

Since $\bar{\delta} \bar{\gamma}(b, t)=\bar{\delta}(b)$ and $d_{V}(b, t)=0 \Rightarrow \bar{\delta}(b)=d_{I[1]}(t)=-d_{I[2]}(t)$ the morphism $\bar{\delta} \bar{\gamma}$ is trivial in homology. 


\section{Finite deformation functors}

The notion of homotopy equivalence of morphisms works well for finitely generated or nilpotent dg-algebras but it seems quite restrictive for general differential algebras. In the set-up of projective limits of nilpotent dg-algebras it is useful to introduce a notion which arises naturally when we consider projective limits in homotopy categories.

Definition 4.1. Let $f, g: S \rightarrow R$ be morphisms of differential algebras, with $R$ complete for the $R$-adic topology (i.e. $\left.R=\lim R / R^{n}\right)$; we shall say that $f$ is prohomotopy equivalent to $g$ if the morphisms $f_{n}, g_{n}: S \rightarrow \overleftarrow{R} \rightarrow R / R^{n}$, composition of $f, g$ with the natural projection $R \rightarrow R / R^{n}$, are homotopy equivalent for every $n$.

It is clear that the relation defined in 4.1 is an equivalence relation, if $R$ is nilpotent it is the same of the usual homotopy equivalence.

If $f, g: S \rightarrow R$ are prohomotopy equivalent morphisms of quasismooth complete differential algebras then for every $A \in \mathbf{C}$ and every $\phi: R \rightarrow A$ there exists a factorization $\phi: R \rightarrow R / R^{n} \rightarrow A, n>>0$, and therefore the composition $\phi f, \phi g$ are homotopy equivalent. This means that $f$ and $g$ induces the same morphism between deformation functors $[R,-] \rightarrow[S,-]$.

Another aspect of prohomotopy is the following

Theorem 4.2. Every complete quasismooth differential algebra is prohomotopy equivalent to a minimal complete quasismooth differential algebra. Two minimal complete quasismooth algebras are prohomotopy equivalent if and only if they are isomorphic.

Proof As in the nongraded case, a morphism $f: S \rightarrow R$ of complete quasismooth differential algebras is an isomorphism if and only if $f_{2}: S / S^{2} \rightarrow R / R^{2}$ is an isomorphism. Since the cohomology of $\left(S / S^{2}\right)^{\vee}$ is isomorphic, up to a shift, to the tangent space of the deformation functor $[S,-]$, if $f$ is a prohomotopy equivalence then $[S,-] \simeq[R,-]$ and $f_{2}$ is a quasiisomorphism; this proves that every prohomotopy equivalence of minimal complete quasismooth differential algebras is an isomorphism.

Let $(R, d)$ be a fixed complete quasismooth differential algebra and let $d_{1}^{1}: R / R^{2} \rightarrow R / R^{2}$ be the differential induced by $d$. Let $R / R^{2}=H \oplus W$ be a decomposition with $W$ acyclic complex and $d_{1}^{1}(H)=0$; fix homogeneous basis $\left\{h_{j}\right\}$ of $H$ and $\left\{v_{i}, w_{i}\right\}$ of $W$ such that $d_{1}^{1}\left(v_{i}\right)=w_{i}$ for every $i$. Since $d\left(v_{i}\right)=w_{i}+\phi_{i}$ for some $\phi_{i} \in R^{2}$, up to the analytic change of coordinates

$$
h_{j} \rightarrow h_{j}, \quad v_{i} \rightarrow v_{i}, \quad w_{i} \rightarrow d v_{i}
$$

it is not restrictive to assume $d W \subset W$. In particular the ideal $(W) \subset R$ is a differential ideal and the quotient $S=R /(W)$ is a minimal complete quasismooth differential algebra.

Our aim is to show that the projection $R \stackrel{\pi}{\longrightarrow} S$ is a prohomotopy equivalence; as a first step we prove that there exists a right inverse $\gamma: S \rightarrow R$ and then we prove that $\gamma \pi: R / R^{n} \rightarrow$ $R / R^{n}$ is homotopic to the identity for every $n>0$.

Since $R$ is complete it is sufficient to find a sequence of morphisms $\gamma_{n}: S \rightarrow R / R^{n}, n \geq 2$, such that $\gamma_{2}: S \rightarrow H=S / S^{2} \rightarrow R / R^{2}=H \oplus W$ is the natural inclusion and the diagrams

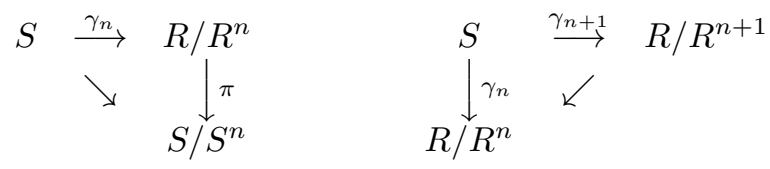

are commutative.

We note that the natural morphism

$$
R / R^{n+1} \rightarrow R / R^{n} \times_{S / S^{n}} S / S^{n+1}
$$


is an acyclic small extension for every $n \geq 2$. According to 2.3 we may define inductively $\gamma_{n+1}: S \rightarrow R / R^{n+1}$ as a lifting of

$$
\left(\gamma_{n}, p_{n+1}\right): S \rightarrow R / R^{n} \times_{S / S^{n}} S / S^{n+1}
$$

where $p_{n}: S \rightarrow S / S^{n}$ denotes the projection.

Up to a change of coordinates of the form $h_{j} \rightarrow \gamma \pi h_{j}, v_{i} \rightarrow v_{i}, w_{i} \rightarrow w_{i}$ we can assume that $S \subset R$ is the complete subalgebra generated by $H$. The homotopies

$$
H_{n}: R \rightarrow R / R^{n}[t, d t], \quad h_{j} \rightarrow h_{j}, \quad v_{i} \rightarrow v_{i} \otimes t, \quad w_{i}=d v_{i} \rightarrow d\left(v_{i} \otimes t\right)
$$

gives the required prohomotopy between $\gamma \pi$ and the identity.

Let's denote by $K$ (quasismooth) the category of quasismooth complete dg-algebras with morphisms up to prohomotopy equivalence; then there exists the prorepresantability functor

$$
(K(\text { quasismooth }))^{o p p} \rightarrow \text { Def, } \quad S \rightarrow[S,-]
$$

Definition 4.3. A deformation functor $F: \mathbf{C} \rightarrow$ Set is called finite if its tangent space $T F[1]$ has finite total dimension.

Definition 4.4. A complete quasismooth dg-algebra $R$ is called finite if $[R,-]$ is a finite deformation functor; i.e. if the graded vector space $H_{*}\left(R / R^{2}\right)$ has finite total dimension.

Note that a minimal complete quasismooth dg-algebra $R$ is finite if and only if $\mathbb{K} \oplus R$ is Noetherian. If $\mathbf{D e f}^{b} \subset$ Def denotes the full subcategory of finite deformation functors and $K$ (quasismooth) $^{b} \subset K$ (quasismooth) denotes the full subcategory of finite complete quasismooth dg-algebras we have

Theorem 4.5. The restriction of the prorepresentability functor

$$
\vartheta:\left(K(\text { quasismooth })^{b}\right)^{o p p} \rightarrow \text { Def }^{b}
$$

is an equivalence of categories.

Proof After theorem 4.2 it is not restrictive to assume that every complete quasismooth dg-algebra is minimal and finite.

STEP 1: $\vartheta$ is injective on morphisms.

Let $f, g: S \rightarrow R$ be morphisms of dg-algebras inducing the same natural transformation of functors $[R,-] \rightarrow[S,-]$; if $\pi_{n}: R \rightarrow R / R^{n}$ denotes the natural projection then $\pi_{n} f, \pi_{n} g: S \rightarrow$ $R / R^{n}$ are homotopic maps and then $f$ and $g$ are prohomotopic by definition.

STEP 2: $\vartheta$ is surjective on morphisms.

Let $S, R$ be finite complete minimal quasismooth dg-algebras and $\alpha:[R,-] \rightarrow[S,-]$ be a natural transformation of functors. In the same notation of Step 1 we will construct recursively a coherent sequence of dg-algebra morphisms $\gamma_{n}: S \rightarrow R / R^{n}$ such that $\left[\gamma_{n}\right]=\alpha\left[\pi_{n}\right] \in$ $\left[S, R / R^{n}\right]$. The resulting inverse limit $\gamma=\lim _{\leftarrow} \gamma_{n}: S \rightarrow R$ will be a morphism of dg-algebras inducing $\alpha$.

Assume $\gamma_{1}, \ldots, \gamma_{n}$ as above are constructed for a fixed $n$ and let $f: S \rightarrow R / R^{n+1}$ be a representative of $\alpha\left[\pi_{n+1}\right]$. Denoting by $p: R / R^{n+1} \rightarrow R / R^{n}$ the projection there exists a homotopy $H: S \rightarrow R / R^{n}[t, d t]$ such that $H_{0}=p f, H_{1}=\gamma_{n}$. As $\operatorname{Hom}_{\mathbf{D G A}}(S,-)$ is a predeformation functor, the argument of Step 3 in the proof of 2.8 shows that there exists a homotopy $K: S \rightarrow R / R^{n+1}[t, d t]$ such that $p K=H$ and $K_{0}=f$; it is therefore sufficient define $\gamma_{n+1}=K_{1}$. 
STEP 3: $\vartheta$ is surjective on isomorphism classes.

Let $F$ be a deformation functor with finite dimensional tangent space $T F[1]$. By 3.3 we need to prove that there exists a minimal complete quasismooth dg-algebra $(R, d)$ and a natural transformation of functors $\xi:[R,-] \rightarrow F$ which is an isomorphism on tangent spaces.

For every integer $i$, let $V_{-i}$ be the dual of the vector space $T F[1]^{i}$, then $V=\oplus_{i} V_{i}=$ $\operatorname{Hom}^{*}(T F[1], \mathbb{K})$ is a finite dimensional graded vector space. Thinking of $V$ as an object in $\mathbf{D G} \cap \mathbf{C}$, we have by 3.2 a natural isomorphism

$$
F(V)=\oplus_{i}\left(T F[1]^{i} \otimes V_{-i}\right)=\operatorname{Hom}_{\mathbf{D G}}(T F[1], T F[1]) .
$$

We denote by $\xi_{2} \in F(V)$ the elements corresponding to the identity on $T F[1]$. Again by 3.2 for every $I \in \mathbf{D G} \cap \mathbf{C}$, the map

$$
\xi_{2}:[V, I] \rightarrow F(I), \quad[f] \rightarrow f\left(\xi_{2}\right)
$$

is bijective. Let $R$ be the inverse limit of $R / R^{n+1}=\oplus_{i=1}^{n}\left(\odot^{i} V\right)$; we want to define two coherent sequences, the first of square zero differentials $d_{n}: R / R^{n} \rightarrow R / R^{n}[1]$ with $d_{2}=0$ and the second of elements $\xi_{n} \in F\left(R / R^{n}, d_{n}\right)$ lifting $\xi_{2}$. This will give a structure of minimal complete quasismooth dg-algebra on $R=\lim R / R^{n}$ and the required natural transformation

$$
[R,-] \rightarrow F, \quad[f: R \rightarrow A] \rightarrow f\left(\xi_{n}\right), n>>0 .
$$

By induction assume there are defined $d_{n}, \xi_{n}$, since $\xi_{n}$ lifts $\xi_{2}$, by 3.12 and 3.10 there exists a unique square-zero differential $d_{n+1}: R / R^{n+1} \rightarrow R / R^{n+1}$ [1] lifting $d_{n}$ and such that the obstruction to lifting $\xi_{n}$ to $\left(R / R^{n+1}, d_{n+1}\right)$ vanishes.

Corollary 4.6. For every finite deformation functor $F: \mathbf{C} \rightarrow$ Set, the induced functor $[F]: K(\mathbf{C}) \rightarrow$ Set is prorepresentable by a quasismooth minimal complete differential algebra $R$. If $T^{i} F=0$ for every $i \leq 0$ then $\mathbb{K} \oplus R$ is the algebra of functions of a formal pointed noetherian semismooth dg-scheme.

Proof Since prorepresentable means representable by a projective limit the proof comes immediately from Theorem 4.5 .

\section{Differential graded coalgebras and $L_{\infty}$-algebras}

Definition 5.1. A cocommutative coassociative $\mathbb{Z}$-graded coalgebra is the data of a graded vector space $C=\oplus_{n \in \mathbb{Z}} C^{n}$ and of a coproduct $\Delta: C \rightarrow C \otimes C$ such that:

- $\Delta$ is a morphism of graded vector spaces.

- $\left(\right.$ coassociativity) $\left(\Delta \otimes I d_{C}\right) \Delta=\left(I d_{C} \otimes \Delta\right) \Delta: C \rightarrow C \otimes C \otimes C$.

- (cocommutativity) $T \Delta=\Delta$.

A morphism of coalgebras is a morphism of graded vector spaces that commutes with coproducts.

By coassociativity we can define the iterated coproduct $\Delta^{n}=\left(I d_{C} \otimes \Delta^{n-1}\right) \Delta: C \rightarrow \otimes^{n} C$. A coassociative coalgebra $(C, \Delta)$ is called nilpotent if $\Delta^{n}=0$ for $n>>0$. 
Example 5.2. The reduced symmetric coalgebra is by definition $\overline{S(V)}=\oplus_{n>0} \odot^{n} V$, with the coproduct:

$$
\Delta\left(v_{1} \odot \ldots \odot v_{n}\right)=\sum_{r=1}^{n-1} \sum_{\sigma \in S(r, n-r)} \epsilon(\sigma)\left(v_{\sigma(1)} \odot \ldots \odot v_{\sigma(r)}\right) \otimes\left(v_{\sigma(r+1)} \odot \ldots \odot v_{\sigma(n)}\right) .
$$

An easy computation about unshuffles shows that the map $N: \overline{S(V)} \rightarrow \overline{T(V)}$ is a morphism of coalgebras.

The coalgebra $\overline{S(V)}$ is coassociative, cocommutative without counity. Note also that $V=$ $\operatorname{ker} \Delta$. We shall say that a subcoalgebra $C \subset \overline{S(V)}$ is homogeneous if $C=\oplus_{n}\left(C \cap \odot^{n} V\right)$.

Following [16] we denote by $C(V)$ the coalgebra $(\overline{S(V[1])}, \Delta)$.

Definition 5.3. Let $(C, \Delta)$ be a coalgebra. A morphism $d: C \rightarrow C[n]$ of graded vector spaces is called a coderivation of degree $n$ if

$$
\Delta d=\left(d \otimes I d_{C}+I d_{C} \otimes d\right) \Delta .
$$

$A$ coderivation $d$ is called a codifferential if $d^{2}=d \circ d=0$.

More generally if $\theta: C \rightarrow D$ is a morphism of coalgebras, a morphism of graded vector spaces $d: C \rightarrow D[n]$ is called a coderivation (with respect to $\theta$ ) if

$$
\Delta_{D} d=(d \otimes \theta+\theta \otimes d) \Delta_{C} .
$$

Note that, by the rule of sign, if $d: C \rightarrow C[n]$ is a morphism of graded vector spaces then $\left(I d_{C} \otimes d\right)(x \otimes y)=(-1)^{n \bar{x}} x \otimes d(y)$. The coderivations of degree $n$ of a coalgebra $C$ form a vector space denoted by $\operatorname{Coder}^{n}(C, C)$.

Lemma 5.4. Let $\theta_{1}, \theta_{2}: C \rightarrow D$ be morphisms of coalgebras and assume that there exists a graded subspace $B \subset C$ such that $\Delta_{C}(C) \subset B \otimes B$ and $\theta_{1}(b)=\theta_{2}(b)$ for every $b \in B$. Then every coderivation $C \rightarrow D[n]$ with respect to $\theta_{1}$ is also a coderivation with respect to $\theta_{2}$.

Proof Evident.

The reduced symmetric coalgebra is a free object in the the category of graded, locally nilpotent, cocommutative, coassociative coalgebras. In fact holds the following:

Proposition 5.5. Let $V$ be a graded vector space, $\pi: \overline{S(V)} \rightarrow V$ the projection and $C$ a cocommutative coalgebra which is union of nilpotent subcoalgebras.

1. The composition with $\pi$ gives a bijection between the set of coalgebra morphisms $\theta: C \rightarrow$ $\overline{S(V)}$ and morphisms of graded vector spaces $m: C \rightarrow V$. The inverse is given by the formula

$$
\theta=\sum_{n=1}^{\infty} \frac{1}{n !} m_{n} \Delta_{C}^{n-1}
$$

where $m_{n}\left(c_{1} \otimes \ldots \otimes c_{n}\right)=m\left(c_{1}\right) \odot \ldots \odot m\left(c_{n}\right)$.

2. For a fixed coalgebra morphism $\theta: C \rightarrow \overline{S(V)}$, the composition with $\pi$ gives an isomorphism

$$
\operatorname{Coder}^{n}(C, \overline{S(V)}) \rightarrow \operatorname{Hom}_{\mathbf{G}}(C, V[n])
$$


Proof 1) is proved in 22, Prop. 4.2 on page 285].

2) follows easily by 1 ) in view of the following observation: consider the coalgebra $C \oplus C[-n]$ with coproduct

$$
\Delta\left(c_{1}+c_{2}[-n]\right)=\Delta\left(c_{1}\right)+\Delta\left(c_{2}\right)[-n]+T\left(\Delta\left(c_{2}\right)[-n]\right) .
$$

(In the above formula we used the natural isomorphism $(C \otimes C)[-n]=C[-n] \otimes C)$. Then $d: C[-n] \rightarrow \overline{S(V)}$ is a coderivation (with respect to $\theta$ ) if and only if $\theta+d: C \oplus C[-n] \rightarrow \overline{S(V)}$ is a morphism of coalgebras.

Definition 5.6. By a dg-coalgebra we intend a triple $(C, \Delta, d)$, where $(C, \Delta)$ is a graded coassociative cocommutative coalgebra and $d: C \rightarrow C[1]$ is a codifferential of degree 1 . The category of dg-coalgebras, where morphisms are morphisms of coalgebras commuting with codifferentials, is denoted by DGC.

Example 5.7. Given $A=\oplus A_{i} \in \mathbf{C}$ let $C=A^{\vee}$ be its graded dual: i.e. $C=\oplus C^{i}$, where $C^{i}=\operatorname{Hom}_{\mathbb{K}}\left(A_{-i}, \mathbb{K}\right)$. We denote by $<,>: C \times A \rightarrow \mathbb{K}$ the induced pairing.

The pairing $\left\langle c_{1} \otimes c_{2}, a_{1} \otimes a_{2}>=<c_{1}, a_{1}><c_{2}, a_{2}>\right.$ gives a natural isomorphism $C \otimes C=$ $(A \otimes A)^{\vee}$ commuting with the twisting maps $T$ and we may define $\Delta$ as the transpose of the multiplication map $\mu: A \otimes A \rightarrow A$.

Then $(C, \Delta)$ is a coassociative cocommutative coalgebra. Note that $C$ is nilpotent if and only if $A$ is nilpotent.

Defining the codifferential in $C$ as the transpose of the differential in $A$ we get a finite dimensional nilpotent dg-coalgebra.

Definition 5.8. Let $V$ be a graded vector space; a codifferential of degree 1 over the symmetric coalgebra $C(V)=\overline{S(V[1])}$ is called an $L_{\infty}$ structure on $V$. The dg-coalgebra $(C(V), Q)$ is called an $L_{\infty}$-algebra.

A $L_{\infty}$-algebra $(C(V), Q)$ is called minimal if $Q_{1}^{1}=0$.

Let $(C(V), Q)$ be a $L_{\infty}$-algebra and let $Q_{j}^{i}: \odot^{j}(V[1]) \rightarrow \odot^{i}(V[1])[1]$ be the composition of the codifferential $Q$ with the inclusion $\odot^{j}(V[1]) \subset C(V)$ and the projection $C(V)[1] \rightarrow$ $\odot^{i}(V[1])[1]$; by Proposition 5.5 the codifferential $Q$ is uniquely determined by the linear maps $Q_{j}^{1}, j>0$ by the explicit formula (cf. [21])

$$
Q\left(v_{1} \odot \ldots \odot v_{n}\right)=\sum_{k=1}^{n} \sum_{\sigma \in S(k, n-k)} \epsilon(\sigma) Q_{k}^{1}\left(v_{\sigma(1)} \odot \ldots \odot v_{\sigma(k)}\right) \odot v_{\sigma(k+1)} \odot \ldots \odot v_{\sigma(n)} .
$$

In particular we have $Q_{j}^{i}=0$ for every $i>j$ and then the subcoalgebras $\oplus_{i=1}^{r}\left(\odot^{i} V\right)$ are preserved by $Q$.

We define a functor $M C_{V}: \mathbf{C} \rightarrow$ Set by setting:

$$
M C_{V}(A)=\operatorname{Hom}_{\mathbf{D G C}}\left(A^{\vee}, C(V)\right)
$$

We use the following terminology: for every coalgebra $C$ and every linear map $\phi: C \rightarrow$ $C(V)[n]$ we denote by $\phi^{i}: C \rightarrow \odot^{i}(V[1])[n]$ the composition of $\phi$ with the projection $C(V)[n] \rightarrow$ $\odot^{i}(V[1])[n]$.

Proposition 5.9. For every $L_{\infty}$-algebra $(C(V), Q)$, the functor $M C_{V}$ is a predeformation functor. 
Proof It is evident that $M C_{V}$ is a covariant functor and $M C_{V}(0)=0$. Let $\alpha: A \rightarrow C$, $\beta: B \rightarrow C$ be morphisms in $\mathbf{C}$, then it is easy to see that the dg-coalgebra $\left(A \times_{C} B\right)^{\vee}$ is the fibered coproduct, in the category DGC, of the morphisms

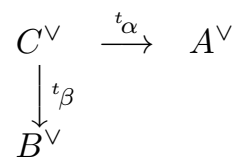

and then

$$
M C_{V}\left(A \times_{C} B\right)=M C_{V}(A) \times_{M C_{V}(C)} M C_{V}(B) .
$$

Let $0 \longrightarrow I \longrightarrow A \longrightarrow B \longrightarrow 0$ be a small acyclic extension in $\mathbf{C}$, we want to prove that $M C_{V}(A) \rightarrow$ $M C_{V}(B)$ is surjective.

We have a dual exact sequence

$$
0 \longrightarrow B^{\vee} \longrightarrow A^{\vee} \longrightarrow I^{\vee} \longrightarrow 0, \quad B^{\vee}=I^{\perp} .
$$

Since $I A=0$ we have $\Delta_{A^{\vee}}\left(A^{\vee}\right) \subset B^{\vee} \otimes B^{\vee}$.

Let $\phi \in M C_{V}(B)$ be a fixed element and $\phi^{1}: B^{\vee} \rightarrow V[1]$; by Proposition $5.5 \phi$ is uniquely determined by $\phi^{1}$. Let $\psi^{1}: A^{\vee} \rightarrow V[1]$ be an extension of $\phi^{1}$, then, by 5.5, $\psi^{1}$ is induced by a unique morphism of coalgebras $\psi: A^{\vee} \rightarrow C(V)$.

The map $\psi d_{A^{\vee}}-Q \psi: A^{\vee} \rightarrow C(V)[1]$ is a coderivation and then, setting $h=\left(\psi d_{I^{\vee}}-Q \psi\right)^{1} \in$ $\operatorname{Hom}_{\mathbf{G}}\left(I^{\vee}, V[2]\right)$, we have that $\psi$ is a morphism of dg-coalgebras if and only if $h=0$.

Note that $\psi^{1}$ is defined up to elements of $\operatorname{Hom}_{\mathbf{G}}\left(I^{\vee}, V[1]\right)=(V[1] \otimes I)^{0}$ and, since $\Delta_{A^{\vee}}\left(A^{\vee}\right) \subset$ $B^{\vee} \otimes B^{\vee}, \psi^{i}$ depends only by $\phi$ for every $i>1$. Since $I$ is acyclic and $h d_{I^{\vee}}+Q_{1}^{1} h=0$ there exists $\xi \in \operatorname{Hom}_{\mathbf{G}}\left(I^{\vee}, V[1]\right)$ such that $h=\xi d_{I^{\vee}}-Q_{1}^{1} \xi$ and then $\theta^{1}=\psi^{1}-\xi$ induces a dg-coalgebra morphism $\theta: A^{\vee} \rightarrow C(V)$ extending $\phi$.

Definition 5.10. Let $(C(V), Q)$ be a $L_{\infty}$-algebra and let $\operatorname{Def}_{V}=M C_{V}^{+}$be the deformation functor associated to the predeformation functor $M C_{V}$ (Theorem 2.8). We shall call $\mathrm{Def}_{V}$ the deformation functor associated to the $L_{\infty}$-algebra $(C(V), Q)$.

A morphism of $L_{\infty}$-algebras $C(V) \rightarrow C(W)$ induces in the obvious way a natural transformation $M C_{V} \rightarrow M C_{W}$ and then, by Theorem 2.8, a morphism $\operatorname{Def}_{V} \rightarrow \operatorname{Def}_{W}$.

For every $A \in \mathbf{C}$ there exists an algebra structure on $\operatorname{Hom}_{\mathbf{G}}\left(A^{\vee}, C(V)\right)$ induced by the natural isomorphism $\operatorname{Hom}_{\mathbf{G}}\left(A^{\vee}, C(V)\right)=(C(V) \otimes A)^{0}$; the product of $f, g: A^{\vee} \rightarrow C(V)$ is given by $f g=\mu(f \otimes g) \Delta_{A^{\vee}}$, where $\mu: C(V) \otimes C(V) \rightarrow C(V)$ is the multiplication of the reduced symmetric algebra $C(V)=\overline{S(V[1])}$. According to 5.5 a morphism $f: A^{\vee} \rightarrow C(V)$ is a morphism of coalgebras if and only if there exist $m \in \operatorname{Hom}_{\mathbf{G}}\left(A^{\vee}, V[1]\right)$ such that

$$
f=\exp (m)-1=\sum_{n=1}^{\infty} \frac{1}{n !} m^{n}
$$

Lemma 5.11. Let $Q$ be a $L_{\infty}$ structure on $V$ and $m \in(V[1] \otimes A)^{0}$. Then $\exp (m)-1$ belongs to $M C_{V}(A)=\operatorname{Hom}_{\mathbf{D G C}}\left(A^{\vee}, C(V)\right)$, via the isomorphism $\operatorname{Hom}_{\mathbf{G}}\left(A^{\vee}, C(V)\right)=(C(V) \otimes A)^{0}$, if and only if

$$
I d_{V[1]} \otimes d_{A}(m)=Q^{1} \otimes I d_{A}(\exp (m)-1)=\sum_{n=1}^{\infty} \frac{1}{n !} Q_{n}^{1} \otimes I d_{A}\left(m^{n}\right) .
$$


Proof Denote $\theta=\exp (m)-1: A^{\vee} \rightarrow C(V)$, then $\theta d_{A^{\vee}}, Q \theta$ are coderivations and then $\theta d_{A^{\vee}}=Q \theta$ if and only if

$$
I d_{V[1]} \otimes d_{A}(m)=\left(\theta d_{A^{\vee}}\right)^{1}=Q^{1} \theta=Q^{1} \otimes I d_{A}(\exp (m)-1) .
$$

The above lemma allow to define $M C_{V}(A)$ for every nilpotent dg-algebra $A$; therefore we can define, as in [16, 4.5.2], the deformation functor $D e f_{V}$ as the quotient of $M C_{V}$ by the equivalence relation given by: $x, y \in M C_{V}(A), x \sim y$ if and only if there exists a solution $z \in M C_{V}(A[t, d t])$ such that $x=z_{t=0}, y=z_{t=1}$. The equivalence of this definition with $M C_{V}^{+}$follows immediately from the equality $A[t, d t]=\cup_{\epsilon>0} V \otimes A[t, d t]_{\epsilon}$, which implies that $M C_{V}(A[t, d t])$ is the direct limit of $M C_{V}\left(A[t, d t]_{\epsilon}\right)$, and the explicit construction of $M C_{V}^{+}$ given in Theorem 2.8.

Lemma 5.12. Let $(C(V), Q)$ be a $L_{\infty}$-algebra and consider $V$ as a differential graded vector space with differential $Q_{1}^{1}$. Then for every $A \in \mathbf{C} \cap \mathbf{D G}$ we have

1. $M C_{V}(A)=\operatorname{Hom}_{\mathbf{D G}}\left(A^{\vee}, V[1]\right)=Z^{1}(V \otimes A)$,

2. $\operatorname{Def}_{V}(A)=H^{1}(V \otimes A)$ and then $T^{i} \operatorname{Def}_{V}=H^{i}(V)$.

Proof Since $A^{2}=0$ we have $\Delta_{A \vee}=0$ and then $\phi^{i}=0$ for every $i \geq 2$ and every morphism of coalgebras $\phi: A^{\vee} \rightarrow C(V)$ : this proves 1). The proof of 2) follows from 1) and Lemma 2.10.

The following result is well known (cf. [16], 18):

Proposition 5.13. Given a graded vector space $V$, there exists a bijection between the set of $D G L A$ structures $(V, d,[]$,$) and the set of L_{\infty}$-algebra structures $(C(V), Q)$ such that $Q_{j}^{1}=0$ for every $j \geq 3$.

Explicitly the bijection is given by $d(w)[1]=-Q_{1}^{1}(w[1])$ and $\left[w_{1}, w_{2}\right]=(-1)^{\overline{w_{1}}} Q_{2}^{1}\left(w_{1}[1] \odot\right.$ $\left.w_{2}[1]\right)$

An easy computation shows that for every DGLA $(V, d,[]$,$) the definitions 2.16$ and 5.10 give the same functor $M C_{V}$, cf. [16].

\section{The Lie algebra structure on $T F$}

Let $F$ be a deformation functor, $T=T F$. For every finite dimensional graded subspace $V \subset T[1]$ we have $F\left(V^{\vee}\right)=\operatorname{Hom}_{\mathbf{G}}(V, T[1])$ and therefore there exists a distinguished element $\xi_{V} \in F\left(V^{\vee}\right)$ corresponding to the inclusion $V \subset T[1]$.

Proposition 6.1. There exists a unique morphism of graded vector spaces $Q_{2}^{1}: \odot^{2}(T[1]) \rightarrow$ $T[2]$ with the following property:

For every pair of finite dimensional graded subspaces $V \subset H \subset T[1]$ such that $Q_{2}^{1}\left(\odot^{2} V\right) \subset H$ consider the subcoalgebra $A=H \oplus\left(\odot^{2} V\right) \subset C(T)$ endowed with the codifferential

$$
\left(\begin{array}{cc}
0 & Q_{2}^{1} \\
0 & 0
\end{array}\right): H \oplus\left(\odot^{2} V\right) \rightarrow\left(H \oplus\left(\odot^{2} V\right)\right)[1] .
$$

Then $\xi_{H}$ lifts to $F\left(A^{\vee}\right)$. 
Proof Let $V \subset T[1]$, taking the dual of the exact sequence

$$
0 \longrightarrow V \longrightarrow V \oplus\left(\odot^{2} V\right) \longrightarrow \odot^{2} V \longrightarrow 0
$$

we get a small extension of finite dimensional nilpotent graded algebras

$$
0 \longrightarrow\left(\odot^{2} V\right)^{\vee} \longrightarrow V^{\vee} \oplus\left(\odot^{2} V\right)^{\vee} \longrightarrow V^{\vee} \longrightarrow 0 .
$$

Let $o b_{V}: F\left(V^{\vee}\right)=\operatorname{Hom}_{\mathbf{G}}(V, T[1]) \rightarrow F\left(\left(\odot^{2} V\right)^{\vee}[1]\right)=\operatorname{Hom}_{\mathbf{G}}\left(\odot^{2} V, T[2]\right)$ be the associated obstruction map and let $q_{V}=o b_{V}\left(\xi_{V}\right)$. Note that $q_{V}$ is, up to sign, the primary obstruction map introduced in 3.9. By base change, for every $V \subset H \subset T[1], q_{V}$ is the obstruction to lifting $\xi_{H}$ to $F\left(H^{\vee} \oplus\left(\odot^{2} V\right)^{\vee}\right)$. In particular if the image of $q_{V}\left(\odot^{2} V\right) \subset H[1]$, by Lemma 3.10 the codifferential

$$
\left(\begin{array}{cc}
0 & -q_{V} \\
0 & 0
\end{array}\right): H \oplus\left(\odot^{2} V\right) \rightarrow\left(H \oplus\left(\odot^{2} V\right)\right)[1]
$$

is the unique codifferential in $A=H \oplus \odot^{2} V$ such that $\xi_{H}$ lifts to $F\left(A^{\vee}\right)$. Glueing all the $-q_{V}$, where $V$ range over all finite dimensional graded subspaces of $T[1]$, we get the required $\operatorname{map} Q_{2}^{1}$.

Theorem 6.2. Let $Q: C(T) \rightarrow C(T)[1]$ be the linear map defined by

$$
Q\left(v_{1} \odot \ldots \odot v_{n}\right)=\sum_{\sigma \in S(2, n-2)} \epsilon(\sigma) Q_{2}^{1}\left(v_{\sigma(1)} \odot v_{\sigma(2)}\right) \odot v_{\sigma(3)} \odot \ldots \odot v_{\sigma(n)} .
$$

Then $Q$ is a codifferential and then $(C(T), Q)$ is a minimal $L_{\infty}$ algebra.

Proof According to standard formula for $L_{\infty}$ algebras, see e.g. [21], the map $Q$ is a coderivation of degree 1 and $Q^{2}=0$ if and only if $Q^{2}\left(\odot{ }^{3} T[1]\right)=0$.

Let $V \subset W \subset Z \subset T[1]$ be finite dimensional subspaces such that $Q\left(\odot^{2} V\right) \subset W[1]$ and $Q\left(\odot{ }^{2} W\right) \subset Z[1]$. Then $B=Z \oplus\left(\odot^{2} W\right) \oplus\left(\odot^{3} V\right)$ is a subcoalgebra of $C(T)$ such that $Q(B) \subset B$. Let $\delta:\left(Z \oplus\left(\odot^{2} W\right)\right)^{\vee} \rightarrow\left(\odot^{3} V\right)^{\vee}[2]$ be the transpose of $Q^{2}$, by Proposition 3.12 we have $\delta F\left(\left(Z \oplus \odot^{2} W\right)^{\vee}\right)=0$. Since the element $\xi_{Z}$ lifts to some $\xi \in F\left(\left(Z \oplus\left(\odot^{2} W\right)\right)^{\vee}\right)$ and $\delta(\xi)=0$ we also have that $\delta=0$ and then also $Q^{2}(B)=0$.

The $L_{\infty}$ algebra $(C(T), Q)$ is clearly minimal and, since $Q_{j}^{1}=0$ for every $j \geq 3$ it comes from a graded Lie algebra $(T,[]$,$) .$

\section{Geometric deformation functors}

In this section we are interested, given a deformation functor $F$, to find sufficient conditions for the existence of a $L_{\infty}$-algebra $V$ such that $F=\operatorname{Def}_{V}$. A straightforward generalization of the transpose of the proof of Corollary 4.6 in the set-up of coalgebras shows that, if $T^{i} F$ is finite dimensional for every $i$, then there exists a structure of minimal $L_{\infty}$-algebra on $T=T F$ such that $F=\operatorname{Def}_{T}$.

Here we consider a special class of functors that, in our opinion, contains all the "interesting" deformation functors.

By extending the analogue definition for classical deformation functors [6] we shall say that a predeformation functor $G$ is homogeneous if for every pair of morphisms $A \rightarrow C$, $B \rightarrow C$ in the category $\mathbf{C}$ we have

$$
G\left(A \times_{C} B\right)=G(A) \times_{G(C)} G(B) .
$$


We say that a deformation functor $F$ if geometric if $F=G^{+}$for some homogeneous predeformation functor $G$. It is not clear to us whether every deformation functor is geometric; in any case we strongly suspect that all the concrete example of deformation functors having some algebro-geometric interest have this property. All the examples of deformation functors considered in this paper are geometric.

Theorem 7.1. Let $F$ be a geometric deformation functor and $T=T F$. Then there exists a minimal $L_{\infty}$ structure on $T$ and an isomorphism of functors $\xi: \operatorname{Def}_{T} \rightarrow F$.

Before proving the theorem we need some preparatory results.

Definition 7.2. Let $(C(V), Q)$ be a $L_{\infty}$ algebra and $\left\{v_{i}\right\}$ a fixed homogeneous basis of $V[1]$. We shall say that a dg-subcoalgebra $A \subset C(V)$ is standard if $A$ has a basis of monomials $v_{i_{1}} \odot \ldots \odot v_{i_{r}}$; in particular every standard $d g$-subcoalgebra is homogeneous.

Lemma 7.3. Let $(C(V), Q)$ be a minimal $L_{\infty}$-algebra, then it is the union of its finite dimensional standard dg-subcoalgebras (with respect to a fixed homogeneous basis of $V[1]$ ).

Proof For every finite dimensional subspace $W \subset C(V)$ there exist $n>0$ and a finite dimensional standard subspace $H_{n}$ such that $W \subset \oplus_{i=1}^{n}\left(\odot^{i} H_{n}\right)$. For every sequence of finite dimensional standard subspaces

$$
0 \subset H_{n} \subset H_{n-1} \subset \ldots \subset H_{1} \subset V[1]
$$

we can consider the standard subcoalgebra $C=\oplus_{i=1}^{n}\left(\odot^{i} H_{i}\right)$. The condition $Q(C) \subset C$ is equivalent to

$$
Q_{j}^{1}\left(\odot^{j} H_{s}\right) \subset H_{s+1-j}, \quad \forall s \geq j \geq 1 .
$$

Assume we have the subspaces $H_{n}, \ldots, H_{r}$ satisfying the above conditions. If $r>1$ then we may take $H_{r-1}$ any finite dimensional standard subspace containing $\sum_{j \geq 2} Q_{j}^{1}\left(\odot^{j} H_{r+j-2}\right)$.

Lemma 7.4. Let $(C(V), Q)$ be a fixed minimal $L_{\infty}$-algebra and $\left\{v_{i}\right\}$ a fixed homogeneous basis of $V[1]$; denote by $C_{n}$ the dg-subcoalgebra $\oplus_{i=1}^{n} \odot^{i}(V[1])$. For a fixed integer $n \geq 2$ let $\mathcal{A}$ be the set of finite dimensional standard dg-subcoalgebras of $C_{n} ; \mathcal{A}$ is a directed set with the ordering given by inclusions. Let $f: \mathcal{A} \rightarrow$ Set be a contravariant functor such that:

1. if $A \subset C_{n-1}$, then $f(A)=\{*\}$ is the one-point set.

2. $f(A+B) \rightarrow f(A) \times_{f(A \cap B)} f(B)$ is bijective.

3. $f(A) \neq \emptyset$ for every $A \in \mathcal{A}$.

Then

$$
f(\mathcal{A}):=\operatorname{liminv}_{A \in \mathcal{A}} f(A) \neq \emptyset .
$$

Proof We shall say that a subset $\Lambda \subset \mathcal{A}$ is saturated if:

1. If $A \subset C_{n-1}$ then $A \in \Lambda$.

2. If $A, B \in \mathcal{A}, A \subset B$ and $B \in \Lambda$ then $A \in \Lambda$.

3. If $A, B \in \Lambda$ then $A+B \in \Lambda$. 
For every saturated $\Lambda$ let $f(\Lambda)$ be the set of coherent sequences $x \in \prod_{A \in \Lambda} f(A)$.

Let $\mathcal{B}$ the set of all pairs $(\Lambda, x)$ with $\Lambda \subset \mathcal{A}$ saturated, $f(\Lambda) \neq \emptyset$ and $x \in f(\Lambda)$. On $\mathcal{B}$ there exists a natural ordering: $\left(\Lambda_{1}, x_{1}\right) \leq\left(\Lambda_{2}, x_{2}\right)$ if and only if $\Lambda_{1} \subset \Lambda_{2}$ and $x_{2}$ extends $x_{1}$. Clearly $\mathcal{B} \neq \emptyset$ and every chain in $\mathcal{B}$ has an upper bound. By Zorn's lemma there exists a maximal element $(\Lambda, x) \in \mathcal{B}$; we want to prove that $\Lambda=\mathcal{A}$.

Assume $\Lambda \neq \mathcal{A}$ and let $A \in \mathcal{A}-\Lambda$ such that the dimension of $A$ is minimum. Necessarily we must have $\operatorname{dim}_{\mathbb{K}}(A)=\operatorname{dim}_{\mathbb{K}}\left(A \cap C_{n-1}\right)+1, A \cap B \subset C_{n-1}$ and $f(A+B)=f(A) \times f(B)$ for every $B \in \Lambda$. Let $\Lambda^{\prime}$ be the union of $\Lambda$ and $\{A+B \mid B \in \Lambda\}$. Choose an element $x_{A} \in f(A)$, then it is uniquely determined an element $x_{A+B} \in f(A+B)$ for every $B \in \Lambda$. We need to prove that, if $C \subset A+B$ then either $C=A+B$ or $C \in \Lambda^{\prime}$ and $x_{A+B}$ extends $x_{C}$.

Assume $C \neq A+B$, since $A, B, C$ are standard we have $C=(A \cap C)+(B \cap C)$. If $A \subset C$ then $C=A+(B \cap C) \in \Lambda^{\prime}$, since $x_{B}$ extends $x_{B \cap C}$ also $x_{A+B}$ extends $x_{C}$. If $A \cap C \neq A$ then $C \cap A \in \Lambda, C \cap A \subset C_{n-1}$ and $f(C)=f(C \cap B)$; since $x_{A+B}$ extends $x_{B}$ it also extends $x_{C}$.

Remark 7.5. It is clear that Lemma 7.4 also works if the codifferential $Q$ is defined only in the subcoalgebra $C_{n}$.

Proof of 7.1 Denote by $S_{n}=\oplus_{i=1}^{n}\left(\odot^{i}(T[1])\right)$. We recall that for every graded vector space $I \in \mathbf{C} \cap \mathbf{G}$ we have

$$
F(I)=\operatorname{Hom}_{\mathbf{G}}\left(I^{\vee}, T[1]\right)=\operatorname{Hom}_{\mathbf{D G C}}\left(I^{\vee}, S_{1}\right),
$$

where $S_{1}$ is endowed with the codifferential $d_{1}=0$. In particular for every finite dimensional standard subspace $J \subset T[1]$ we have $F\left(J^{\vee}\right)=\operatorname{Hom}_{\mathbf{G}}(J, T[1])$ : we denote by $\xi_{J} \in F\left(J^{\vee}\right)$ the element corresponding to the inclusion $J \hookrightarrow T[1]$ and by

$$
\xi_{1}=\left(\xi_{J}\right) \in \prod_{J \subset T[1]} F\left(J^{\vee}\right) .
$$

Let $G$ be a homogeneous predeformation functor such that $F=G^{+}$and let $\pi: G \rightarrow F$ the projection.

We now construct recursively a sequence $\left(d_{n}, \eta_{n}\right)$ such that for every $n \geq 1$

$A_{n}: d_{n}: S_{n} \rightarrow S_{n-1}[1] \subset S_{n}[1]$ is a codifferential extending $d_{n-1}$.

$B_{n}: \eta_{n} \in \operatorname{liminv} F\left(G^{\vee}\right)$, where:

1. the limit is taken over all the finite dimensional standard dg-subcoalgebras of $\left(S_{n}, d_{n}\right)$.

2. $\eta_{1}$ is a lifting of $\xi_{1}$.

3. $\eta_{n}$ is a lifting of $\eta_{n-1}$ if $n \geq 2$.

For every standard subspace $J \subset T[1]$ let $f(J) \subset G\left(J^{\vee}\right)$ be the set of liftings of $\xi_{J}$; the functor $f$ satisfies the hypothesis of Lemma 7.4 and then there exists $\eta_{1}$ that lifts $\xi_{1}$. Let $n \geq 2$ be a fixed integer and assume are given $\left(d_{1}, \eta_{1}\right), \ldots,\left(d_{n-1}, \eta_{n-1}\right)$ satisfying $A_{i}, B_{i}$, $i=1, \ldots, n-1$.

Let $A \subset S_{n}$ be a fixed standard finite dimensional subcoalgebra and denote $B=A \cap S_{n-1}$, $J=A \cap S_{1}$. Possibly enlarging $B$, we may assume that $d_{n-1}(B) \subset B$ and $d_{n-1}$ can be extended to a coderivation on $A$; dualizing we get a small extension of finite dimensional graded nilpotent algebras

$$
0 \longrightarrow B^{\perp} \longrightarrow A^{\vee} \longrightarrow B^{\vee} \longrightarrow 0
$$


such that $B^{\perp}$ and $B^{\vee}$ have respective differential equal to 0 and ${ }^{t} d_{n-1}$.

Let $\xi_{B}=\pi\left(\eta_{B}\right) \in F\left(B^{\vee}\right)$ be the projection of $\xi_{n-1}=\pi\left(\eta_{n-1}\right)$. Since $\xi_{B}$ lifts $\xi_{J}$ and $J^{\vee}=B^{\vee} /\left(B^{\vee}\right)^{2}$ the map

$$
\left[B^{\vee}, B^{\perp}[2]\right] \rightarrow F\left(B^{\perp}[2]\right), \quad[f] \rightarrow f\left(\xi_{B}\right)
$$

is injective and, by Proposition 3.12, there exists a differential on $A^{\vee}$ making the exact sequence $0 \rightarrow B^{\perp} \rightarrow A^{\vee} \rightarrow B \rightarrow 0$ a small extension in $\mathbf{C}$.

Let $o \in F\left(B^{\perp}[1]\right)=\operatorname{Hom}_{\mathbf{G}}(A / B, T[2])$ be the obstruction to the lifting of $\xi_{B}$ to $F\left(A^{\vee}\right)$; possibly enlarging $B$, we may suppose that $o \in \operatorname{Hom}_{\mathbf{G}}(A / B, J[1])$ and then there exists an unique morphism $\phi: B^{\vee} \rightarrow B^{\perp}[1]$ such that $o=\phi\left(\xi_{B}\right)$. By Lemma 3.10 there exists an unique differential on $A$ making $0 \rightarrow B^{\perp} \rightarrow A^{\vee} \rightarrow B \rightarrow 0$ a small extension in $\mathbf{C}$ and such that $\xi_{B}$ lifts to $F\left(A^{\vee}\right)$. The transpose of this differential is a codifferential in $A$.

Clearly all these codifferentials in the finite dimensional homogeneous subcoalgebras of $S_{n}$ can be glued together and we get a global codifferential $d_{n}: S_{n} \rightarrow S_{n-1}[1]$.

For every $A \subset S_{n}$ finite dimensional standard dg-subcoalgebra let

$$
f(A)=\left\{x \in G\left(A^{\vee}\right) \mid x \text { is a lifting of } \eta_{A \cap S_{n-1}}\right\} .
$$

By Lemma 2.9 and the choice of $d_{n}, f(A) \neq \emptyset$ for every $A$. The functor $f$ satisfies the hypothesis of Lemma 7.4 and then $\operatorname{liminv} f(A) \neq \emptyset$. By construction every $\eta_{n} \in \operatorname{liminv} f(A)$ satisfies the condition $B_{n}$.

The sequence $\eta=\left(\eta_{n}\right)$ gives a natural transformation $\xi: M C_{T} \rightarrow G$ by the rule:

$$
\operatorname{Hom}_{\mathbf{D G C}}\left(A^{\vee}, C(T)\right) \ni \alpha \rightarrow \eta(f)={ }^{t} \alpha\left(\eta_{D}\right) \in F(A),
$$

where $D$ is a finite dimensional standard dg-subcoalgebra of $C(T)$ such that $\alpha\left(A^{\vee}\right) \subset D$. By construction the induced morphism $\operatorname{Def}_{T}=M C_{T}^{+} \rightarrow F$ is an isomorphism on tangent spaces and then, according to Corollary 3.3 it is an isomorphism of functors.

\section{References}

[1] S. Barannikov, M. Kontsevich: Frobenius manifolds and formality of Lie algebras of polyvector fields. Internat. Math. Res. Notices 4 (1998) 201-215.

[2] S. Barannikov: Generalized periods and mirror symmetry in dimension $n>3$. math.AG/9903124.

[3] A. Canonaco: $L_{\infty}$-algebras and quasi-isomorphisms. In: Seminari di Geometria Algebrica 1998-1999 Scuola Normale Superiore (1999).

[4] I. Ciocan-Fontanine, M.M. Kapranov: Derived Quot Schemes math.AG/9905174.

[5] I. Ciocan-Fontanine, M.M. Kapranov: Derived Hilbert Schemes math.AG/0005155.

[6] B.Fantechi, M. Manetti: Obstruction calculus for functors of Artin rings, I. Journal of Algebra 202 (1998) 541-576.

[7] B. Fantechi, M. Manetti: On the $T^{1}$-lifting theorem. J. Alg. Geom. 8 (1999) 31-39.

[8] W.M. Goldman, J.J. Millson: The deformation theory of representations of fundamental groups of compact Kähler manifolds. Publ. Math. I.H.E.S. 67 (1988) 43-96.

[9] W.M. Goldman, J.J. Millson: The homotopy invariance of the Kuranishi space. Ill. J. Math. 34 (1990) 337-367. 
[10] M. Grassi: $L_{\infty}$-algebras and differential graded algebras, coalgebras and Lie algebras. In: Seminari di Geometria Algebrica 1998-1999 Scuola Normale Superiore (1999).

[11] M. Grassi: Graded manifolds, flat families and the global case of the formality theorem In: Seminari di Geometria Algebrica 1998-1999 Scuola Normale Superiore (1999).

[12] P.H. Griffiths, J.W. Morgan: Rational Homotopy Theory and Differential Forms. Birkhäuser Progress in Mathematics 16 (1981).

[13] V. Hinich: DG coalgebras as formal stacks. math.AG/9812034.

[14] M. Kapranov: Injective resolutions of BG and derived moduli spaces of local systems. J. Pure Appl. Algebra 155 (2001) 167-179.

[15] M. Kontsevich: Enumeration of rational curves via torus action. In Moduli Space of Curves (R. Dijkgraaf, C. Faber, G. van der Geer Eds) Birkhäuser (1995) 335-368.

[16] M. Kontsevich: Deformation quantization of Poisson manifolds, I, q-alg/9709040.

[17] M. Kontsevich: Topics in algebra-deformation theory. Notes (1994):

[18] T. Lada, M. Markl: Strongly homotopy Lie algebras. Comm. Algebra 23 (1995) 21472161.

[19] T. Lada, J. Stasheff: Introduction to sh Lie algebras for physicists. Int. J. Theor. Phys. 32 (1993) 1087-1104.

[20] M. Manetti: Deformation theory via differential graded Lie algebras. In: Seminari di Geometria Algebrica 1998-1999 Scuola Normale Superiore (1999).

[21] M. Penkava: $L_{\infty}$-algebras and their cohomology. q-alg/9512014.

[22] D. Quillen: Rational homotopy theory. Ann. of Math. 90 (1969) 205-295.

[23] M. Schlessinger: Functors of Artin rings. Trans. Amer. Math. Soc. 130 (1968) 208-222.

[24] M. Schlessinger, J. Stasheff: The Lie algebra structure of tangent cohomology and deformation theory. J. Pure Appl. Algebra 38 (1985) 313-322.

Marco Manetti

Dipartimento di Matematica "G. Castelnuovo", Università di Roma "La Sapienza",

Piazzale Aldo Moro 5, I-00185 Roma, Italy.

manetti@mat.uniroma1.it, http://www.mat.uniroma1.it/people/manetti/ 